\title{
DYNAMICS OF THE SHARP EDGES OF BROAD PLANETARY RINGS
}

\author{
Joseph M. Hahn ${ }^{1}$, Joseph N. Spitale ${ }^{2}$, and Carolyn C. Porco ${ }^{2}$ \\ ${ }^{1}$ Space Science Institute, 10500 Loring Drive, Austin, TX 78750, USA; jhahn@ spacescience.org \\ ${ }^{2}$ CICLOPS, Space Science Institute, 4750 Walnut Street, Suite 205, Boulder, CO 80301, USA; spitale@ pirl.lpl.arizona.edu, carolyn@ ciclops.org \\ Received 2008 December 29; accepted 2009 April 7; published 2009 June 15
}

\begin{abstract}
This paper describes a model of a broad planetary ring whose sharp edge is confined by a satellite's $m$ th Lindblad resonance (LR). This model uses the streamline formalism of Borderies et al. to calculate the ring's internal forces, namely, ring gravity, pressure, and viscosity. The model also allows for the possibility of a drag force that can affect small ring particles directly, and large ring particles indirectly via collisions with the small. The model calculates the streamlines' forced eccentricities $e$, their longitudes of periapse $\tilde{\omega}$, and the surface density $\sigma$ throughout the perturbed ring. This model is then applied to the outer edge of Saturn's B ring, which is maintained by an $m=2$ inner LR with the satellite Mimas. A suite of ring models are used to illustrate how a ring's perturbed state depends on the ring's physical properties: its surface density, its viscosity, the ring particles' dispersion velocity, and the strength of the hypothetical drag force. A comparison of simulations with the outer B ring's observed properties suggests that the ring's surface density there is $10 \lesssim \sigma \lesssim 280 \mathrm{gm} \mathrm{cm}^{-2}$ in the ring's outermost $\sim 40 \mathrm{~km}$. The ring's sharp edge identifies the site where the ring's viscous torque precisely counterbalances the perturbing satellite's gravitational torque on the ring. However, an examination of several seemingly conventional viscous B ring models shows that they all fail, by wide margins, to balance these torques at the ring's outer edge. This is partly due to the ring's self-gravity, which tends to reduce forced eccentricities near the resonance. But this is also due to the fact that a viscous ring tends to be nearly peri-aligned with the satellite. Both effects conspire to reduce the satellite's torque on the ring, which in turn makes the ring's edge more difficult to maintain. Nonetheless, the following shows that a torque balance can still be achieved in a viscous B ring, but only in an extreme case where the ratio of the ring's bulk/ shear viscosities satisfy $v_{b} / v_{s} \sim 10^{4}$. However, if the dissipation of the ring's forced motions is instead dominated by a weak drag force, then the satellite can exert a much stronger torque across a wider annulus in the ring, which can successfully counterbalance the ring's viscous torque there. We also show how this streamline model can be adapted to study other interesting ring phenomena, such as narrow eccentric ringlets and nonlinear spiral density waves.
\end{abstract}

Key words: planets: rings

\section{INTRODUCTION}

The outer edge of Saturn's main B ring is confined by an $m=2$ inner Lindblad resonance (ILR) with the satellite Mimas, while the outer edge of the main A ring is confined by $m=7$ ILRs with the coorbital satellites Janus and Epimetheus (Porco et al. 1984). Ring particles orbiting near a resonance execute $m$ radial excursions, or epicycles, which gives a ring's edge a scalloped, $m$-lobed appearance. The ring particles' epicyclic amplitude $R_{m}$, which is the half-amplitude of the particles' radial excursions, is obviously governed in part by the mass of the perturbing satellite. However, the ring's internal forcesself-gravity, pressure, and viscosity-also play a role here by tempering the ring's response to the satellite's resonant gravitational perturbations. Consequently, modeling these rings in a way that accounts for all of these perturbations, and then comparing those models to observations of the ring's edge, should allow one to assess the relative importance of a ring's various internal forces. This activity will also allow one to make estimates of, or place limits on, the ring's physical parameters, such as its surface density $\sigma$, viscosity $v$, and the ring particles' dispersion velocity $c$. Also note that small ring particles are susceptible to drag forces-plasma drag, Poynting-Robertson drag, and/or the Yarkovsky effect-whose significance can also be assessed by this kind of modeling.

The epicyclic amplitudes of the outer A and B rings are quite small, $R_{m} \sim 10$ 's of $\mathrm{km}$, while the ring's radii are $r \sim 10^{5} \mathrm{~km}$, so the ring particles' noncircular motions are only $\sim 0.01 \%$ in a fractional sense. The smallness of those motions also makes any time-dependent ring models, such as $N$-body, $\mathrm{SPH}$, or hydrodynamic treatments, very difficult, due to the very many particles or hydrodynamic cells needed to simulate the ring-edge's entire circumference. Also keep in mind that one does not know the ring's equilibrium state in advance, so simulations of a ring's time-evolution would initially be dominated by transient motions that can easily mask the ring's small forced motions. Consequently, time-dependent models must also evolve the system until the ring's dissipation has damped out those transients, which can also cost a lot of CPU time.

Semianalytic methods instead provide a much more efficient and illuminating method for studying perturbed planetary rings. These methods are enabled by the streamline concept, which is a very powerful tool for studying planetary rings. A streamline is the epicyclic path that is traced by numerous ring particles that all have a common semimajor axis. A planetary ring can then be thought of as a sum of many such streamlines. This streamline approach also allows one to calculate the forces that any one streamline exerts on any one ring particle. Summing over all the forces exerted by all of the ring's streamlines, and inserting those forces into Newton's equations of motion, then provides a model that can be used to calculate the perturbed ring's equilibrium state.

Streamlines were first used to show how a narrow ringlet's self-gravity can counter the differential precession that occurs when orbiting an oblate planet (Goldreich \& Tremaine 1979). A streamline approach was also used to show how viscosity helps a satellite maintain a planetary ring's sharp edge (Borderies 
et al. 1982), and streamlines were used to examine how the gapembedded satellite Pan maintains the Encke gap in Saturn's A ring (Borderies et al. 1989). Streamlines are also used to study nonlinear spiral density waves (Borderies et al. 1986; Longaretti $\&$ Borderies 1986). Evidently, the streamline concept is a very general tool that can be used to study a variety of interesting ring phenomena. And in the following, we use a streamline formalism to examine in detail how a satellite's $m$ th ILR can disturb as well as maintain the sharp outer edges of Saturn's main rings.

Section 2 reviews the subject in detail, and derives all of the equations that will be needed to assemble a comprehensive model of a broad planetary ring whose edge is confined by a satellite's $m$ th Lindblad resonance. However, a reader who is uninterested in the many details and derivations can skip ahead to Section 3, which examines a suite of B ring models that illustrate how the perturbed ring's state-its epicyclic amplitude, its orientation, and its surface density variationsall depend on the ring's physical properties: $\sigma, v$, and $c$. This section also shows how observations can be used to infer a ring's physical properties, which for the B ring are quite unknown. Section 4 then discusses some important side issues, while Section 5 summarizes our main findings.

\section{EQUATION OF MOTION}

This section derives the equation of motions that will be used to calculate the motion of an orbiting ring particle while it is perturbed by an orbiting satellite and the planetary ring. The particle's position vector $\mathbf{r}(t)$ evolves over time $t$ according to Newton's second law of motion,

$$
\ddot{\mathbf{r}}=\frac{d^{2} \mathbf{r}}{d t^{2}}=-\nabla \Phi+\mathbf{a},
$$

where $\Phi(\mathbf{r})=\Phi_{p}+\Phi_{s}$ is the total gravitational potential that is due to the central planet $\Phi_{p}$ and satellite $\Phi_{s}$, and $\mathbf{a}$ is the acceleration of the particle that is due to the forces exerted by the planetary ring. For simplicity, the following assumes that the satellite's orbit is circular and coplanar with the ring plane, but these results are unchanged if the satellite's orbit is instead slightly noncircular or inclined. Polar coordinates will also be used, where $\mathbf{r}=(r, \theta)$ with $r(t)$ being the particle's distance from the planet's center, and $\theta(t)$ its longitude measured relative to some $\hat{\mathbf{x}}$ axis, with the $\hat{\mathbf{z}}$ axis perpendicular to the orbital plane. Solutions to the equation of motion (1) are then obtained after Fourier expanding the perturbations that are acting on the orbiting ring particle.

\subsection{Fourier Expansions of the Perturbations}

Planet-centered coordinates will be used, so the satellite's gravitational potential has direct and indirect parts that are

$$
\Phi_{s}(r, \theta)=-\frac{G m_{s}}{\left|\mathbf{r}-\mathbf{r}_{\mathbf{s}}\right|}+\frac{G m_{s}}{r_{s}^{3}} \mathbf{r} \cdot \mathbf{r}_{s},
$$

where $G$ is the gravitational constant, $m_{s}$ is the satellite's mass, and $\mathbf{r}_{s}=\left(a_{s}, \theta_{s}\right)$ is its position vector in polar coordinates, with $a_{s}$ being the satellite's semimajor axis and $\theta_{s}$ its longitude. A Fourier expansion of that potential is

$$
\Phi_{s}(r, \theta)=\frac{1}{2} \phi_{s}^{0}(r)+\mathfrak{R} e\left(\sum_{m=1}^{\infty} \phi_{s}^{m}(r) e^{\mathrm{i} m\left(\theta-\theta_{s}\right)}\right)
$$

where the potential's Fourier amplitudes $\phi_{s}^{m}$ are

$$
\begin{aligned}
\phi_{s}^{m}(r) & =\frac{1}{\pi} \int_{-\pi}^{\pi} \Phi_{s}(r, \varphi) \cos (m \varphi) d \varphi \\
& =-\frac{G m_{s}}{a_{s}}\left[b_{1 / 2}^{(m)}(\beta)-\beta \delta_{m 1}\right],
\end{aligned}
$$

where $\varphi=\theta-\theta_{s}$ is the particle's longitude relative to the satellite's, and the Laplace coefficient

$$
b_{s}^{(m)}(\beta)=\frac{2}{\pi} \int_{0}^{\pi} \frac{\cos (m \varphi) d \varphi}{\left(1+\beta^{2}-2 \beta \cos \varphi\right)^{s}}
$$

is a function of the ratio $\beta=r / a_{s}$. The Kronecker delta $\delta_{m 1}$ in Equation (4) is due to the indirect part of the potential in Equation (2), which only contributes to the $m=1$ part of the satellite's gravity.

The acceleration that the ring exerts on the particle is $\mathbf{a}(r, \theta)=a_{r} \hat{\mathbf{r}}+a_{\theta} \hat{\theta}$, where $a_{r}$ and $a_{\theta}$ are the radial and tangential components. A Fourier expansion of those accelerations will also have the form

$$
\begin{aligned}
& a_{r}(r, \theta)=A_{r}^{0}(r)+\Re e\left(\sum_{m=1}^{\infty} A_{r}^{m}(r) e^{\mathrm{i} m\left(\theta-\theta_{s}\right)}\right) \\
& a_{\theta}(r, \theta)=A_{\theta}^{0}(r)+\Re e\left(\sum_{m=1}^{\infty} A_{\theta}^{m}(r) e^{\mathrm{i} m\left(\theta-\theta_{s}\right)}\right) .
\end{aligned}
$$

These Fourier expansions are convenient since each of the $m \geqslant 1$ terms in Equation (3) corresponds to Lindblad resonances that are all spatially segregated. Consequently, when solving the equation of motion for the particle's motion, we only need to retain a single $m$ th term in the expression for $\Phi_{s}$. This is also true for the ring's internal accelerations $a_{r}$ and $a_{\theta}$, since they are excited by the satellite's $m$ th resonant perturbation of the ring. In light of this, we write

$$
\begin{gathered}
\Phi_{s}(r, \theta) \simeq \phi_{s}^{m}(r) e^{\mathrm{i} m\left(\theta-\theta_{s}\right)} \\
a_{r}(r, \theta) \simeq A_{r}^{0}(r)+A_{r}^{m}(r) e^{\mathrm{i} m\left(\theta-\theta_{s}\right)} \\
a_{\theta}(r, \theta) \simeq A_{\theta}^{0}(r)+A_{\theta}^{m}(r) e^{\mathrm{i} m\left(\theta-\theta_{s}\right)}
\end{gathered}
$$

with the $\Re e()$ notation dropped henceforth, so it is to be understood that one is to preserve only the real parts of the following equations. Also note that the axisymmetric part of the satellite's potential, $\phi_{s}^{0}$, was omitted from Equation (7a), since it is convenient to combine it with the planet's potential, $\Phi_{p} \rightarrow \Phi_{p}+\phi_{s}^{0}$, which is also axisymmetric. Lastly, note that $\phi_{s}^{m}, A_{r}^{0}$, and $A_{\theta}^{0}$ are all real, while the $A_{r}^{m}$ and $A_{\theta}^{m}$ can be complex.

\subsection{Motion Near a Lindblad Resonance}

The radial and tangential parts of the particle's equation of motion are

$$
\begin{gathered}
\ddot{r}-r \dot{\theta}^{2}=-\frac{\partial \Phi}{\partial r}+a_{r} \\
\frac{1}{r} \frac{d}{d t}\left(r^{2} \dot{\theta}\right)=-\frac{1}{r} \frac{\partial \Phi}{\partial \theta}+a_{\theta}
\end{gathered}
$$


when Equation (7) is inserted into Equation (1). Similar equations are solved in Goldreich \& Tremaine (1982) for an isolated particle that does not experience a perturbation from the ring (i.e., $\mathbf{a}=0$ ), so the following solution to the more general $\mathbf{a} \neq 0$ problem will use a strategy and notation similar to that given in Goldreich \& Tremaine (1982). A ring particle's orbit will be nearly circular, so its trajectory has the form

$$
r(t)=r_{0}+r_{1}(t) \quad \text { and } \quad \theta(t)=\theta_{0}+\Omega_{0} t+\theta_{1}(t),
$$

where the constant $r_{0}$ is the particle's mean distance from the planet, $\Omega_{0}$ is its mean angular velocity about the planet, and $\theta_{0}$ is an arbitrary phase. Since the perturbing accelerations a and $-\nabla \Phi_{s}$ are all small compared to the central planet's gravity $-\nabla \Phi_{p}$, the particle's displacements from a purely circular orbit will be small such that $\left|r_{1}\right| \ll r_{0}$ and $\left|\theta_{1}\right| \ll 1$, which then allows the equation of motion to be linearized.

The satellite's orbital angular velocity is $\Omega_{s}$, so its longitude is $\theta_{s}=\Omega_{s} t$ when time $t=0$ is chosen to be the time when it traverses the $\hat{\mathbf{x}}$ axis. This also means that the particle's relative longitude that appears in Equation (7) is $m\left(\theta-\theta_{s}\right)=m \theta_{0}+\omega_{m} t+m \theta_{1}$ where $\omega_{m}(r)=m\left(\Omega_{0}-\Omega_{s}\right)$ is the particle's Doppler-shifted forcing frequency. But this quantity is usually needed only to lowest order in the small angles, so

$$
m\left(\theta-\theta_{s}\right) \simeq m \theta_{0}+\omega_{m} t
$$

The particle's specific angular momentum is $h=r^{2} \dot{\theta}$, and the time evolution of that quantity is obtained from Equation (8b) with $\dot{\theta}=h / r^{2}$ :

$$
\frac{d h}{d t}=-\frac{\partial \Phi}{\partial \theta}+r a_{\theta}=-\mathrm{i} m \phi_{s}^{m} e^{\mathrm{i} m\left(\theta-\theta_{s}\right)}+r A_{\theta}^{0}+r A_{\theta}^{m} e^{\mathrm{i} m\left(\theta-\theta_{s}\right)} .
$$

The first term is the specific torque that the satellite exerts on the ring particle, while the other terms are the specific torques that are due to the ring's internal forces. Evidently, the total torque $d h / d t$ is the sum of secular (i.e., nonoscillatory) terms like $r_{0} A_{\theta}^{0}$ plus other oscillatory terms. In light of this, write $h(t)=h_{0}(t)+h_{1}(t)$ where $h_{0}=r_{0}^{2} \Omega_{0}$ is the secular part of the particle's specific angular momentum $h(t)$, while $h_{1}(t)$ is the oscillatory part. The secular and oscillatory torques on the particle, $d h_{0} / d t$ and $d h_{1} / d t$, are then

$$
\begin{gathered}
\frac{d h_{0}}{d t} \simeq r_{0} A_{\theta}^{0}+T_{s} \\
\frac{d h_{1}}{d t} \simeq\left(-\mathrm{i} m \phi_{s}^{m}+r_{0} A_{\theta}^{m}\right) e^{\mathrm{i}\left(\theta_{0}+\omega_{m} t\right)}
\end{gathered}
$$

when Equation (11) is written to lowest order in the particle's coordinates, i.e., with $r \simeq r_{0}$ and $m\left(\theta-\theta_{s}\right) \simeq m \theta_{0}+\omega_{m} t$. Note that we have also added to Equation (12a) an additional term $T_{s}$ to represent the secular part of the specific torque that the satellite exerts on the particle; Section 2.6 will show that this second-order term is important only near the ring's sharp edge. And if the ring particle's orbit is to be static such that its mean orbit radius $r_{0}$ is constant, then the secular torque on the particle, $d h_{0} / d t$, must be zero, for otherwise that particle (as well as its neighboring ring particles) would drift radially. Consequently, equilibrium thus requires all particles to satisfy the torque-balance equation, $T_{s}=-r_{0} A_{\theta}^{0}$.

Integrating Equation (12b) with respect to time $t$ provides the oscillatory part of the particle's specific angular momentum,

$$
h_{1}=-\left(\frac{m \phi_{s}^{m}}{\omega_{m}}+\frac{\mathrm{i} r_{0} A_{\theta}^{m}}{\omega_{m}}\right) e^{\mathrm{i}\left(m \theta_{0}+\omega_{m} t\right)} .
$$

Also note that $\left|h_{1}\right|$ is small compared to $h_{0}=r_{0}^{2} \Omega_{0}$. The quantity $h_{1}$ is then used to solve the radial part of the equation of motion, which is

$$
\ddot{r}-\frac{h^{2}}{r^{3}}=-\frac{\partial \Phi_{p}}{\partial r}+A_{r}^{0}+\left(-\frac{\partial \phi_{s}^{m}}{\partial r}+A_{r}^{m}\right) e^{\mathrm{i}\left(m \theta_{0}+\omega_{m} t\right)}
$$

when $\dot{\theta}=h / r^{2}$ and Equations (7) are substituted into Equation (8a). Inserting $r=r_{0}+r_{1}$ and $h=h_{0}+h_{1}$ into the above, Taylor-expanding to first order in the small quantities $r_{1}$ and $h_{1}$, and then inserting $h_{0}=r_{0}^{2} \Omega_{0}$ and Equation (13) into that result then yields

$$
\begin{aligned}
\ddot{r}_{0} & +\left(\frac{\partial \Phi_{p}}{\partial r}-r_{0} \Omega_{0}^{2}-A_{r}^{0}\right)+\ddot{r}_{1}+\left(3 \Omega_{0}^{2}+\frac{\partial^{2} \Phi_{p}}{\partial r^{2}}\right) r_{1} \\
& \simeq\left(-\frac{\partial \phi_{s}^{m}}{\partial r}-\frac{2 m \Omega_{0}}{r_{0} \omega_{m}} \phi_{s}^{m}-\frac{2 \mathrm{i} \Omega_{0}}{\omega_{m}} A_{\theta}^{m}+A_{r}^{m}\right) e^{\mathrm{i}\left(m \theta_{0}+\omega_{m} t\right)}
\end{aligned}
$$

where it is understood that all quantities in the above are to be evaluated at $r=r_{0}$.

The ring particle is assumed to be in torque balance, so $r_{0}$ is constant and $\ddot{r}_{0}=0$. And since the terms in the first set of parentheses in Equation (15) are secular while the remaining terms are oscillatory, that set of parentheses, which is the condition for centrifugal equilibrium, must separately sum to zero, which provides the particle's mean angular velocity $\Omega_{0}=\Omega\left(r_{0}\right)$ where

$$
\Omega^{2}=\frac{1}{r} \frac{\partial \Phi_{p}}{\partial r}-\frac{A_{r}^{0}}{r} .
$$

The constant in the second parentheses in Equation (15) is the particle's epicyclic frequency, $\kappa_{0}=\kappa\left(r_{0}\right)$, where

$$
\kappa^{2}=3 \Omega^{2}+\frac{\partial^{2} \Phi_{p}}{\partial r^{2}} .
$$

Since the ring's radial acceleration $\left|A_{r}^{0}\right|$ is small compared to the central planet's gravity, these angular frequencies are

$$
\Omega \simeq \Omega_{p}\left(1-\frac{A_{r}^{0}}{2 r \Omega_{p}^{2}}\right)
$$

$$
\text { and } \quad \kappa \simeq \kappa_{p}\left(1-\frac{3 A_{r}^{0}}{2 r \kappa_{p}^{2}}\right)
$$

where $\Omega_{p}=\sqrt{r^{-1} \partial \Phi_{p} / \partial r}$ would be the particle's angular velocity if ring forces were absent, and $\kappa_{p}=\sqrt{4 \Omega_{p}^{2}+r \partial \Omega_{p}^{2} / \partial r}$ would be its epicyclic frequency when $A_{r}^{0}=0$. 
The terms on the right of Equation (15) that involve $\phi_{s}^{m}$ are the satellite's forcing function,

$$
\Psi_{s}^{m}(r)=-\frac{\partial \phi_{s}^{m}}{\partial r}-\frac{2 m \Omega}{r \omega_{m}} \phi_{s}^{m},
$$

which accounts for the satellite's radial and tangential forcings. All the coefficients on the right-hand side of Equation (15) will be known as the system's complex forcing function,

$$
\Psi_{c}^{m}(r)=\Psi_{s}^{m}-\frac{2 \mathrm{i} \Omega_{0}}{\omega_{m}} A_{\theta}^{m}+A_{r}^{m} .
$$

Inserting this into the above then casts Equation (15) in its simplest form,

$$
\begin{gathered}
\ddot{r}_{1}+\kappa_{0}^{2} r_{1} \simeq \Psi_{c}^{m}\left(r_{0}\right) e^{\mathrm{i}\left(m \theta_{0}+\omega_{m} t\right)} . \\
\text { 2.3. Single Particle Motion }
\end{gathered}
$$

When the ring's internal forces are absent, i.e., $\mathbf{a}=0$, then $\Psi_{c}^{m}=\Psi_{s}^{m}$ is a constant, and Equation (21) describes a driven simple harmonic oscillator whose solution is

$$
r_{1}(t)=-R_{m} e^{\mathrm{i}\left(m \theta_{0}+\omega_{m} t\right)} .
$$

This solution is examined in Goldreich \& Tremaine (1982), which is summarized here since those results are used throughout this study. Inserting Equation (22) into Equation (21) then yields the particle's epicyclic amplitude $R_{m}$, which is

$$
R_{m}=-\frac{\Psi_{s}^{m}}{D\left(r_{0}\right)}
$$

$$
\text { where } \quad D(r)=\kappa^{2}-\omega_{m}^{2}
$$

is the particle's distance from resonance in frequency-squared units. When a particle is far from a resonance, $|D|$ is of order $\Omega^{2}$, and the particle's epicyclic amplitude $R_{m}$ is negligibly small. However, when the particle is near a resonance, $|D| \ll \Omega^{2}$, and the particle's response to the satellite's resonant forcing is much larger. Exact resonance is the site where $D\left(r_{r}\right)=0$, or where $\kappa=\epsilon \omega_{m}$ with $\epsilon \pm 1$. If the central planet's potential $\Phi_{p}$ were Keplerian, then $\Phi_{p}=-G M_{p} / r$ where $M_{p}$ is the planet's mass and $\kappa=\Omega=\sqrt{G M_{p} / r^{3}}$. Inserting this into the resonance condition $\kappa=\epsilon \omega_{m}=\epsilon m\left(\Omega-\Omega_{s}\right)$ then yields the radius of the Lindblad resonance,

$$
r_{r}=(1-\epsilon / m)^{2 / 3} a_{s},
$$

where $a_{s}$ is the satellite's orbit radius. Resonances having $\epsilon=+1$ are ILRs since they reside interior to the satellite's orbit, while those with $\epsilon=-1$ are outer Lindblad resonances (OLRs). The focus of this work will be on the outer edge of Saturn's main $\mathrm{B}$ ring, which is confined by an ILR with a satellite that orbits exterior to the ring, so $\epsilon=+1$ here. Lastly, note that if the central planet is oblate, then the particle's epicyclic frequency $\kappa$ differs slightly from its angular velocity $\Omega$, so Equation (24) is only approximately true. However, a precise calculation of the resonance location is also given in Section 2.3.1.

For a particle orbiting near a LR it is sufficient to linearize $D(r)$ via $D \simeq x \mathcal{D}$ where $x=\left(r-r_{r}\right) / r_{r}$ is the particle's fractional distance from resonance, and

$$
\mathcal{D}=\left.r \frac{d D}{d r}\right|_{r_{r}}=3 \epsilon(m-\epsilon) \Omega_{0}^{2} .
$$

Inserting Equation (4) into Equation (19) and noting that $\omega_{m} \simeq \epsilon \Omega$ near a $\mathrm{LR}$ also provides the satellite's forcing function, which is

$$
\Psi_{s}^{m}\left(r_{r}\right)=\epsilon f_{\epsilon}^{m} \mu_{s} r_{0} \Omega_{0}^{2},
$$

where $\mu_{s}$ is the satellite's mass in units of the central planet's, and $f_{\epsilon}^{m}$ is

$$
f_{\epsilon}^{m}=\epsilon \beta^{2} \frac{\partial b_{1 / 2}^{(m)}}{\partial \beta}+2 m \beta b_{1 / 2}^{(m)}-(2 m+\epsilon) \beta^{2} \delta_{m 1},
$$

which is a positive numerical coefficient that depends on the resonance in question. For instance, this study is interested in Mimas' $m=2$ ILR in the B ring, which has $\epsilon=+1$ and $f_{\epsilon}^{m}=1.500$. Inserting these quantities into Equation (23a) then provides the particle's epicyclic amplitude,

$$
R_{m}=-\frac{f_{\epsilon}^{m} \mu_{s} r_{0}}{3(m-\epsilon) x},
$$

as well as its forced eccentricity

$$
\begin{aligned}
& e=\frac{\left|r_{1}\right|}{r_{0}}=\frac{f_{\epsilon}^{m} \mu_{s}}{3(m-\epsilon)|x|}=\left|\frac{\psi_{s}}{x}\right|, \\
& \text { where } \quad \psi_{s} \equiv \frac{\Psi_{s}^{m}}{\left|r_{0} \mathcal{D}\right|}=\frac{\epsilon f_{\epsilon}^{m} \mu_{s}}{3(m-\epsilon)}
\end{aligned}
$$

is a dimensionless version of the satellite's forcing function.

Note that $R_{m}$ is real and has the opposite sign to $x$. This then allows a parameterization of the particle's radial displacement $r_{1}$ in terms of its longitude $\theta$,

$$
r_{1}(\theta) \simeq \operatorname{sgn}(x)\left|R_{m}\right| \cos m\left(\theta-\theta_{s}\right),
$$

when Equation (10) is utilized. Also note that the magnitude of the particle's radial excursion $\left|r_{1}\right|$ is maximal when it arrives at that satellite's longitude, $\theta=\theta_{s}$. Consequently, if the particle's orbit lies interior to the resonance with $x<0$, then $r_{1}<0$, so the particle must also be at periapse since $r\left(\theta=\theta_{s}\right)=r_{0}+r_{1}=r_{0}-\left|R_{m}\right|$. Similarly, a particle orbiting exterior to resonance would be at apoapse, $r=r_{0}+\left|R_{m}\right|$, when in conjunction with the satellite. So in summary, orbits interior to an LR are expected to be peri-aligned with the satellite's longitude, while orbits exterior should be apo-aligned.

Interestingly, the outer edge of Saturn's B ring does not behave according to these expectations. Voyager observations revealed that the B ring's outer edge actually lies about $24 \mathrm{~km}$ exterior to the resonance (Porco et al. 1984). This is quite a curiosity, since, if Mimas' $m=2$ ILR is indeed responsible for maintaining this ring's outer edge, then one might expect the ring's outer edge to lie at or just interior to the resonance. Another curiosity is that the ring is peri-aligned with Mimas, whereas one would expect the ring to be apo-aligned if it truly extended beyond the resonance. Porco et al. (1984) suggest that this interesting behavior might be a consequence of the ring's internal forces, which are considered in Section 2.4.

\subsubsection{Resonance Location}

A Lindblad resonance is the site $r=r_{r}$ that satisfies $D\left(r_{r}\right)=0$, which is also where

$$
\kappa\left(r_{r}\right)=\epsilon \omega_{m}\left(r_{r}\right)=\epsilon m\left[\Omega\left(r_{r}\right)-\Omega_{s}\right],
$$


Table 1

Physical Properties of the Saturn-Mimas System ${ }^{\mathrm{a}}$

\begin{tabular}{lc}
\hline \hline Saturn's $G M_{p}$ & $3.79312077 \times 10^{7} \mathrm{~km}^{3} \mathrm{~s}^{-2}$ \\
Saturn's zonal harmonic $J_{2}$ & $1.629071 \times 10^{-2}$ \\
$J_{4}$ & $-9.3583 \times 10^{-4}$ \\
$J_{6}$ & $8.614 \times 10^{-5}$ \\
$J_{8}$ & $-1.0 \times 10^{-5}$ \\
Mimas' angular velocity ${ }^{\mathrm{b}} \Omega_{s}$ & $381.9944522 \mathrm{deg} \mathrm{day}^{-1}$ \\
Mimas' fractional mass $\mu_{s}$ & $6.5969 \times 10^{-8}$ \\
\hline
\end{tabular}

Notes.

${ }^{\mathrm{a}}$ From Jacobson et al. (2006), except where noted otherwise.

${ }^{b}$ As reported by the JPL Solar System Dynamics Web site http://ssd.jpl.nasa.gov/ ?sat_elem on 2008 June 26.

with the frequencies $\Omega$ and $\kappa$ defined by Equations (16) and (17). Those quantities also depend on gradients in the central planet's gravitational potential $\Phi_{p}$, which is

$$
\Phi_{p}(r)=-\frac{G M_{p}}{r}\left[1-\sum_{k=1}^{\infty} P_{2 k}(0) J_{2 k}\left(\frac{r}{R_{p}}\right)^{-2 k}\right]
$$

for bodies orbiting in an oblate planet's equatorial plane, where the $J_{2 k}$ are the planet's zonal harmonics and the $P_{2 k}$ are Legendre polynomials (Murray \& Dermott 1999). All of the Saturnian system's physical constants that are used in the model, $G M_{p}, J_{2 k}$, etc., are listed in Table 1. For instance, solving Equation (31) for the location of Mimas' $m=2$ ILR yields $a_{r}=117,553.71 \mathrm{~km}$ when the standard $R_{p}=60,330 \mathrm{~km}$ is adopted as Saturn's radius. The position $a_{r}$ will be referred to as the nominal resonance position, since exact resonance can be shifted further by the ring's internal forces, which are assessed below.

\subsection{The Ring's Internal Forces}

A ring particle is also subject to additional forces, such as the gravity exerted by the entire ring, and collisions with other ring particles. The cumulative effect of those collisions will be modeled here as if they were due to pressure (Goldreich \& Tremaine 1978). A ring particle is also subject to viscous effects, which could be due to collisions among ring particles (Goldreich \& Tremaine 1982), or perhaps due to transient selfgravitating wakes in the ring (Daisaka et al. 2001) and/or the viscous overstability (Salo et al. 2001). Small ring particles are also susceptible to a number of other drag forces (Goldreich \& Tremaine 1982) that can be accounted for here, too. The following subsections will assess the ring's internal forces, the calculation of which is greatly facilitated by the streamline concept. Borderies et al. (1982) note that Equation (30) also represents a streamline in the ring, which is a closed trajectory that is traced by numerous ring particles having a common semimajor axis $r_{0}$. A broader planetary ring can then be regarded as being comprised of numerous streamlines having distinct semimajor axes $r_{0}$. As Borderies et al. (1985) show, the calculation of the acceleration a that the entire ring exerts on a single particle can be simplified substantially by considering the particle's interaction with individual streamlines. But doing that will first require understanding the relationship between the ring's surface density $\sigma(r, \theta)$ and a streamline's semimajor axis $a=r_{0}$, eccentricity $e$, and its longitude of periapse $\tilde{\omega}$.

\subsubsection{Streamlines}

Equation (20) shows that if the ring's internal forces have a tangential component, then the equation of motion (21) for a ring particle's radial displacement $r_{1}$ is complex, so write

$$
r_{1}(t)=-\mathcal{R}_{m} e^{\mathrm{i}\left(m \theta_{0}+\omega_{m} t\right)} \simeq-\mathcal{R}_{m} e^{\mathrm{i} m\left(\theta-\theta_{s}\right)}=-R_{m} e^{\mathrm{i} m\left(\theta-\theta_{s}-\tilde{\omega}\right)}
$$

where $\mathcal{R}_{m}(a) \equiv R_{m} e^{-\mathrm{i} m \tilde{\omega}}$ is the particle's (or streamline's) complex epicyclic amplitude, and $\tilde{\omega}(a)$ its longitude of periapse, both of which are to be regarded as functions of the streamline's semimajor axis $r_{0}=a$. The particle's total planetocentric distance is then

$$
\begin{aligned}
r(a, \theta) & =a+r_{1} \simeq a-\mathfrak{R} e\left[\mathcal{R}_{m}(a) e^{\mathrm{i} m\left(\theta-\theta_{s}\right)}\right] \\
& =a-R_{m}(a) \cos m\left(\theta-\theta_{s}-\tilde{\omega}\right) .
\end{aligned}
$$

Also identify $e(a)=R_{m} / a$ as the particle's forced eccentricity, with $e$ and $R_{m}$ to be regarded as positive real quantities.

The following will also need the ring particle's longitudinal displacement, $\theta_{1}$, which is obtained by linearizing its total specific angular momentum $h=r^{2} \dot{\theta} \simeq r_{0}^{2} \Omega_{0}+r_{0}^{2} \dot{\theta}_{1}+2 r_{0} \Omega_{0} r_{1}=$ $h_{0}+h_{1}$, so $h_{0}=r_{0}^{2} \Omega_{0}$ as expected and

$\dot{\theta}_{1}=-\frac{2 \Omega_{0} r_{1}}{r_{0}}+\frac{h_{1}}{r_{0}^{2}}=\left(\frac{2 \Omega_{0} \mathcal{R}_{m}}{r_{0}}-\frac{m \Phi_{s}^{m}}{r_{0}^{2} \omega_{m}}+\frac{\mathrm{i} A_{\theta}^{m}}{r_{0} \omega_{m}}\right) e^{\mathrm{i}\left(m \theta_{0}+\omega_{m} t\right)}$,

which is integrated to obtain

$$
\theta_{1} \simeq\left(-\frac{2 \mathrm{i} \epsilon \mathcal{R}_{m}}{r}+\frac{\mathrm{i} m \Phi_{s}^{m}}{r^{2} \Omega^{2}}+\frac{A_{\theta}^{m}}{r \Omega^{2}}\right) e^{\mathrm{i} m\left(\theta-\theta_{s}\right)}
$$

since $\omega_{m} \simeq \epsilon \kappa \simeq \epsilon \Omega$ near an LR. However, the particle's epicyclic amplitude $\mathcal{R}_{m}$ will be large near a resonance, dwarfing the other terms, so

$$
\theta_{1} \simeq \frac{2 \mathrm{i} \epsilon r_{1}}{a}
$$

and

$$
\dot{\theta}_{1} \simeq 2 e \Omega_{0} \exp \left[\operatorname{im}\left(\theta-\theta_{s}-\tilde{\omega}\right)\right]
$$

\subsubsection{Nonlinear Surface Density}

To calculate a perturbed ring's surface density $\sigma$, it is convenient to make the "local" approximation, which assumes that the interparticle forces are exerted primarily by nearby ring particles that lie a small distance $\ell$ away from the perturbed particle, where $\ell \ll a$. Because these perturbing particles reside on streamlines that are close by, one can ignore the streamlines' curvature that only occurs at great distances $\gg \ell$, and instead treat the streamlines as if they were straight wires having a linear mass density $\lambda(a)$ that is essentially constant ${ }^{3}$ about a streamline whose semimajor axis is $a$. Note, however, that perturbations of the ring also changes its surface density $\sigma$, since these streamlines can be compressed or rarefied in the radial direction. To see this, let $\delta m=\lambda \delta \ell$ be the total mass that resides along a segment of a streamline whose tangential length is $\delta \ell$. If the ring were unperturbed, $\delta m=\lambda \delta \ell=\sigma_{0} \delta a \delta \ell$, where $\delta a$ is the streamline's radial width, so $\lambda=\sigma_{0} \delta a$ is the streamline's linear density. If, however, the ring were perturbed, then $\delta m=\lambda \delta \ell=$ $\sigma \delta r \delta \ell$, i.e., $\lambda=\sigma_{0} \delta a=\sigma \delta r$, where $\sigma(a, \theta)$ is the perturbed

\footnotetext{
3 Actually, $\lambda$ varies around the orbital ellipse by a small fractional amount that is of $\mathcal{O}(e) \ll 1$, which may be neglected.
} 
ring's surface density. Consequently, mass conservation requires $\sigma(a, \theta)=\sigma_{0}(a) / J$ where $J(a, \theta)=\delta r / \delta a \rightarrow \partial r / \partial a$ in the limit that the streamline width $\delta a \rightarrow 0$. Thus,

$$
\sigma(a, \theta)=\frac{\sigma_{0}(a)}{J},
$$

where

$$
J(a, \theta)=\frac{\partial r}{\partial a}=\Re e\left[1-\mathcal{R}_{m}^{\prime} e^{\mathrm{i} m\left(\theta-\theta_{s}\right)}\right]
$$

is a measure of the streamlines' degree of compression (Borderies et al. 1985). Note that this quantity is sensitive to gradients in the streamlines' orbit elements, since

$$
\begin{aligned}
\mathcal{R}_{m}^{\prime}=\frac{\partial \mathcal{R}_{m}}{\partial a} & =\left(R_{m}^{\prime}-\mathrm{i} m \frac{\partial \tilde{\omega}}{\partial a} R_{m}\right) e^{-\mathrm{i} m \tilde{\omega}} \\
& =\left[\frac{\partial(a e)}{\partial a}+\mathrm{i} k a e\right] e^{-\mathrm{i} m \tilde{\omega}},
\end{aligned}
$$

where $R_{m}=a e$ and the wavenumber $k \equiv-m(\partial \tilde{\omega} / \partial a)$ is the rate at which the streamline's longitude of periapse varies with semimajor axis $a$. The above can also be written more compactly as

$$
\mathcal{R}_{m}^{\prime}=q e^{\mathrm{i}(\eta-m \tilde{\omega})}
$$

$$
\begin{aligned}
& \text { where } q=\sqrt{\left(e+e^{\prime}\right)^{2}+(k a e)^{2}} \text { and } \\
& \tan \eta=\frac{k a e}{e+e^{\prime}}
\end{aligned}
$$

since $\partial(a e) / \partial a=e+d e / d x \equiv e+e^{\prime}$, so

$$
\begin{aligned}
J=1-q \cos (\phi+\eta) & =1-\Re e\left[q e^{\mathrm{i}(\phi+\eta)}\right] \\
& =\Re e\left\{1-\frac{\partial}{\partial a}[e a \exp (\mathrm{i} \phi)]\right\},
\end{aligned}
$$

where $\phi=m\left(\theta-\theta_{s}-\tilde{\omega}\right)$. The angle $\eta$ indicates when the ring's response to perturbations takes the form of a spiral density wave. When the ring's response is a tightly wrapped wave, $|k a e| \gg\left|e+e^{\prime}\right|$ and so $|\eta| \simeq \pi / 2$, while $|\eta| \ll 1$ when the ring's response is not wavelike.

Note that $q=\left|\mathcal{R}_{m}^{\prime}\right|$ is the nonlinearity parameter of Borderies et al. (1985), who point out that adjacent streamlines cross if their separation $|\delta r|$ exceeds $|\delta a|$ at any longitude $\theta$, which would occur if $q \geqslant 1$. However, previous studies have shown that a ring's internal forces tend to adjust the streamlines so as to avoid crossing (Shu et al. 1985), and we expect $q<1$ here, too.

The ring's response to perturbations is said to be linear when $q \ll 1$. In that case, the fractional variations in the ring's surface density, $\left(\sigma-\sigma_{0}\right) / \sigma_{0} \simeq \mathcal{R}_{m}^{\prime} e^{\mathrm{i} m\left(\theta-\theta_{s}\right)}$, are sinusoidal and of low amplitude. However, when the ring is nonlinear, $q=\left|\mathcal{R}_{m}^{\prime}\right|$ is not small, and large variations in $\sigma(a, \theta)$ can occur. Although those variations in $\sigma(a, \theta)$ would be periodic in longitude $\theta$, Equation (39) shows that they would not be sinusoidal. Lastly, note that Equation (39) also implies that a nonlinear ring's surface density can drop by no more than $50 \%$ since $\left|\mathcal{R}_{m}^{\prime}\right|<1$, which also occurs in the troughs of nonlinear spiral density waves (Shu et al. 1985).

If this study were instead interested in spiral density waves, we would then note that tightly wrapped spiral waves have wavenumbers $|k a| \gg 1$ and amplitudes $e(a) \sim$ constant. In this case, the second term in Equation (41) would dominate over the first, so $\mathcal{R}_{m}^{\prime} \simeq \mathrm{i} k \mathcal{R}_{m}$. This is known as the tight-winding approximation, and it is valid when $|k a e|>>\left|e+e^{\prime}\right|$ such that $\eta \simeq \pm \pi / 2$ in this case. If we were to insert this into Equation (39) and proceed further, we would then arrive at the theory for nonlinear spiral density waves (compare Borderies et al. 1986), which will in fact be considered in a follow-up study.

However, this effort is interested in the motion of particles orbiting near an ILR, most of whom are on the nonwave side of the resonance. Also recall the results of Section 2.3, which suggest that the ring particles there will have similar longitudes of perihelia $\tilde{\omega}$. Since $\tilde{\omega}$ varies slowly with radial distance $x$, the $|k a e|$ term in the above is usually small in comparison to $\left|e+e^{\prime}\right| \simeq\left|e^{\prime}\right|$ term, since the streamline's eccentricities $e(a)$ grow rapidly with proximity to the resonance. Thus, $\mathcal{R}_{m}^{\prime} \simeq(d e / d x) e^{-\mathrm{i} m \tilde{\omega}}, q e^{\mathrm{i} \eta} \simeq e^{\prime}, \eta \simeq 0$ or $\pi$, and $\sigma(a, \theta)=\sigma_{0}(a) / J \simeq \sigma_{0} /\left[1-e^{\prime} \cos m\left(\theta-\theta_{s}-\tilde{\omega}\right)\right]$ in most (but not all) of the scenarios considered here.

Finally, recall that the forced eccentricity of a single isolated ring particle is $e(x)=\psi_{s} / x$ (Equation (29a)), so the threshold for streamline crossing would be $\left|\mathcal{R}_{m}^{\prime}\right|=|d e / d x|=\psi_{s} / x^{2}=$ 1. Solving for $|x| \equiv x_{\mathrm{NL}}$ then provides a rough estimate for the fractional distance from resonance where the ring's surface density variations will be nonlinear, and where the ring's internal forces should be significant (e.g., Borderies et al. 1982):

$$
x_{\mathrm{NL}}=\sqrt{\left|\psi_{s}\right|}=\sqrt{\frac{f_{\epsilon}^{m} \mu_{s}}{3(m-\epsilon)}} .
$$

This study is interested in Mimas' $m=2$ ILR that lies near the B ring's outer edge, which has $\epsilon=1$ and $f_{\epsilon}^{m}=1.50$. The perturbing satellite's mass is $\mu_{s}=6.60 \times 10^{-8}$ Saturn masses, and its $m=2$ resonance lies at $a_{r}=117,553.7 \mathrm{~km}$ from Saturn's center, so $\psi_{s}=3.30 \times 10^{-8}, x_{\mathrm{NL}}=1.8 \times 10^{-4}$, and $\Delta a_{\mathrm{NL}}=x_{\mathrm{NL}} a_{r} \simeq 20 \mathrm{~km}$ is the physical distance from resonance where nonlinear effects play a role in the ring's dynamics. We also note that the outer edge of the A ring is maintained by an $m=7$ ILR with the Janus/Epimetheus coorbital pair. That resonance has $f_{\epsilon}^{m}=9.49, \mu_{s}=3.32 \times 10^{-9}$ (which is the mass of Janus, the larger of the two coorbitals; Porco et al. 2007), and a mean semimajor axis $a_{r}=136,773 \mathrm{~km}$ (Porco et al. 1984), so $\psi_{s}=1.75 \times 10^{-9}, x_{\mathrm{NL}}=4.2 \times 10^{-5}$, and $\Delta a_{\mathrm{NL}} \simeq 6 \mathrm{~km}$. It is over these spatial scales where the ring's internal forces, such as self-gravity, are expected to play an important role.

\subsubsection{Ring Gravity}

In the local approximation, a perturbing streamline is an infinitely long, straight wire having a linear mass density $\lambda\left(a^{\prime}\right)=\sigma_{0}\left(a^{\prime}\right) \delta a^{\prime}$, where $\sigma_{0}\left(a^{\prime}\right)$ is the unperturbed ring's surface density, $a^{\prime}$ its semimajor axis, and $\delta a^{\prime}$ is its radial width in semimajor axis space. The gravitational acceleration that streamline exerts on a nearby particle is $\delta a_{g}=2 G \lambda\left(a^{\prime}\right) / d=$ $2 G \sigma_{0}\left(a^{\prime}\right) \delta a^{\prime} / d$, where $d=r^{\prime}-r^{\prime}$ is the separation between the perturbing streamline, whose planetocentric distance is $r^{\prime}$, and the perturbed ring particle at $r$. Since the streamlines' eccentricities $e(a)$ are small, the streamlines are all nearly parallel, so the gravitational forces that they exert are radial. If the particle's semimajor axis is $a$, then that separation is $d=r^{\prime}-r=a^{\prime}-a-\Re e\left\{\left[R_{m}\left(a^{\prime}\right) e^{-\mathrm{i} m \Delta \tilde{\omega}^{\prime}}-R_{m}(a)\right] e^{\mathrm{i} m\left(\theta-\theta_{s}-\tilde{\omega}\right)}\right\}$ by Equation (34), where $\Delta \tilde{\omega}^{\prime} \equiv \tilde{\omega}\left(a^{\prime}\right)-\tilde{\omega}$. This separation is then 
written more compactly as $d=a\left(x^{\prime}-x\right) \Re e\left[1-\Delta e^{\mathrm{i} m\left(\theta-\theta_{s}-\tilde{\omega}\right)}\right]=$ $a\left(x^{\prime}-x\right)(1-\delta \cos \phi)$, where $\phi=m\left(\theta-\theta_{s}-\tilde{\omega}\right)+\beta$ and

$$
\Delta \equiv \frac{R_{m}\left(a^{\prime}\right) e^{-\mathrm{i} m \Delta \tilde{\omega}^{\prime}}-R_{m}(a)}{a^{\prime}-a}=\frac{e\left(x^{\prime}\right) e^{-\mathrm{i} m \Delta \tilde{\omega}^{\prime}}-e(x)}{x^{\prime}-x} \equiv \delta e^{\mathrm{i} \beta},
$$

where

$$
\tan \beta=\frac{-e\left(x^{\prime}\right) \sin \left(m \Delta \tilde{\omega}^{\prime}\right)}{e\left(x^{\prime}\right) \cos \left(m \Delta \tilde{\omega}^{\prime}\right)-e(x)}
$$

and $\delta=|\Delta|$ is real. The total acceleration that the entire ring exerts on the particle is then

$$
a_{g}(a, \theta)=\int_{\text {ring }} \delta a_{g}=\int_{a_{\text {in }}}^{a_{\text {out }}} \frac{2 G \sigma_{0}\left(a^{\prime}\right) d a^{\prime}}{a\left(x^{\prime}-x\right)(1-\delta \cos \phi)},
$$

where $a_{\text {in }}$ and $a_{\text {out }}$ are the semimajor axes of those streamlines at the ring's innermost and outermost edges.

Next, Fourier-expand the longitude-dependent factor (1 $\delta \cos \phi)^{-1}$ in Equation (47), which is

$$
\begin{aligned}
\frac{1}{1-\delta \cos \phi}= & \frac{1}{\sqrt{1-\delta^{2}}}+\frac{2}{\delta}\left[\left(1-\delta^{2}\right)^{-1 / 2}-1\right] \cos \phi \\
& + \text { other terms, }
\end{aligned}
$$

provided $\delta<1$ such that streamlines do not cross. Those other terms in the above are proportional to $\cos (n \phi)=\cos [n m(\theta-$ $\left.\left.\theta_{s}-\tilde{\omega}\right)+n \beta\right]$, where the integer $n$ takes all values of $n \geqslant 2$. Those other terms represent non-resonant forcings of the ring, and they are negligible since $R_{n m}$ will be small. Inserting the surviving terms in Equation (48) back into Equation (47) then shows that the ring's gravity $a_{g}$ has the desired form of Equation (7b):

$$
\begin{gathered}
A_{g r}^{0}(x)=2 G \int_{a_{\text {in }}}^{a_{\text {out }}} \frac{\sigma_{0}\left(a^{\prime}\right) d x^{\prime}}{\left(x^{\prime}-x\right) \sqrt{1-\delta^{2}}} \\
A_{g r}^{m}(x)=4 G e^{-\mathrm{i} m \tilde{\omega}} \int_{a_{\text {in }}}^{a_{\text {out }}} \frac{\sigma_{0}\left(a^{\prime}\right)\left[\left(1-\delta^{2}\right)^{-1 / 2}-1\right] e^{\mathrm{i} \beta}}{\left(x^{\prime}-x\right) \delta} d x^{\prime} \\
=4 G e^{-\mathrm{i} m \tilde{\omega}} \int_{a_{\text {in }}}^{a_{\text {out }}} \frac{\sigma_{0}\left(a^{\prime}\right)\left[\left(1-\delta^{2}\right)^{-1 / 2}-1\right]}{e\left(x^{\prime}\right) e^{\mathrm{i} m \Delta \tilde{\omega}^{\prime}-e(x)} d x^{\prime}}
\end{gathered}
$$

where the $r$ subscripts indicate that these are radial accelerations.

For numerical work it will be convenient to put Equations (49) in a dimensionless form, which is obtained by dividing by $|a \mathcal{D}|=3(m-\epsilon) a \Omega^{2}$. Also let $s(x)=\sigma(x) / \sigma_{\infty}$ be the ring's fractional surface density, where the constant $\sigma_{\infty}$ is the ring's undisturbed surface density far away from the resonance. Also introduce the so-called normalized disk mass $\mu_{d}=\pi \sigma_{\infty} a^{2} / M_{p}=\pi G \sigma_{\infty} / a \Omega^{2}$, which is a dimensionless measure of the ring's unperturbed surface density that is quite convenient. With these definitions in hand, the dimensionless version of the above accelerations become

$$
\begin{gathered}
\alpha_{g r}^{0}(x)=\frac{A_{g r}^{0}}{|a \mathcal{D}|}=\frac{2 \mu_{d}}{3 \pi(m-\epsilon)} \int_{x_{\text {in }}}^{x_{\text {out }}} \frac{s\left(x^{\prime}\right) d x^{\prime}}{\left(x^{\prime}-x\right) \sqrt{1-\delta^{2}}} \\
\text { and } \quad \alpha_{g r}^{m}(x)=\frac{A_{g r}^{m}}{|a \mathcal{D}|}=\bar{\alpha}_{g r}^{m} e^{-\mathrm{i} m \tilde{\omega}},
\end{gathered}
$$

where $\quad \bar{\alpha}_{g r}^{m}(x)=\frac{4 \mu_{d}}{3 \pi(m-\epsilon)} \int_{x_{\text {in }}}^{x_{\text {out }}} \frac{s\left[\left(1-\delta^{2}\right)^{-1 / 2}-1\right] d x^{\prime}}{e\left(x^{\prime}\right) e^{\mathrm{i} m \Delta \tilde{\omega}^{\prime}}-e(x)}$

with the integration variable $x^{\prime}$ being the fractional distance from the nominal resonance that ranges over the ring's inner and outer boundaries $x_{\text {in }}$ and $x_{\text {out }}$.

\subsubsection{The Hydrodynamic Approximation}

Currently, there is some uncertainty in the rings literature on how to handle the microscopic interactions that occur among ring particles. Those interactions are important, since they control the dynamical heating of a ring as well as the transport of angular momentum through the ring. For many years it had been thought that collisions were the most important particle-particle interaction, and sophisticated models were developed to handle the effects of collisions. For instance, Borderies et al. (1983) solve the Boltzmann equation for the stress tensor that accounts for those interparticle interactions, which yielded a formalism known as the particle-gas model. Borderies et al. (1985) also derive an alternate stress tensor for an incompressible fluidring. We also note that the particle-jam model of Mosqueira \& Estrada (2002) appears to be an intermediate case, since their ring is quasi-incompressible.

However, we now know that self-gravitating wakes also play an important role in stirring dense planetary rings (Daisaka et al. 2001), and that the viscous overstability can be another mechanism for viscous transport in the ring (Salo et al. 2001). In light of the varied and uncertain ways in which ring particles interact on microscopic scales, we have elected to treat these interactions in the simplest way possible, via the hydrodynamic approximation, which assumes that the ring is a Newtonian fluid whose internal stresses manifest themselves via pressure and viscosity. This hydrodynamic approach has been described as "qualitatively acceptable" (Borderies et al. 1982), but we consider it to be the most reasonable and practical approach, given the current uncertainties.

A Newtonian fluid is one where stress is proportional to strain. However, Latter \& Ogilvie (2006) use kinetic theory to show that the influence of wakes and other instabilities in a "dilute" planetary ring can in fact be non-Newtonian. But Latter \& Ogilvie (2006) also show that the results of the kinetic theory do agree with hydrodynamics when the ring is dense and collisions are frequent, as is the outer B ring. It should also be noted that the many spiral density waves that are seen in Saturn's rings provides observational evidence that the hydrodynamic treatment is in fact appropriate, since the damping of linear spiral density waves is well described by simple hydrodynamic viscosity (Tiscareno et al. 2007). Sremcevic et al. (2008) also show that subtle variations seen in these waves' dispersion relation are probably due to hydrodynamic pressure. Both of these observations support the notion that the hydrodynamic approximation is in fact appropriate when studying a planetary ring's large-scale motion, and justify its usage here.

\subsubsection{Particle Collisions and Pressure}

The following will account for the effects of particle collisions in a simple quantitative way, with collisional effects being treated as if they were due to a pressure $p(\mathbf{r}, t)$ that tends to repel particles away from regions of higher density (where collisions will be more frequent and vigorous) and toward 
regions of lower density. To effect this, adopt a barotropic equation of state that assumes that the pressure in the ring can be expressed as the function $p(\mathbf{r}, t)=p(\rho)$ where $\rho(\mathbf{r}, t)$ is the ring's volume density that obeys $c^{2}=d p / d \rho$ where $c$ is the ring particles' dispersion velocity. Then the radial acceleration that an individual ring particle experiences due to collisional pressure is

$$
a_{p}=-\frac{1}{\rho} \frac{d p}{d r}=-\frac{1}{\rho} \frac{d p}{d \rho} \frac{d \rho}{d r}=-\frac{c^{2}}{\rho} \frac{d \rho}{d r}=-\frac{c^{2}}{\sigma} \frac{d \sigma}{d r}
$$

since $\rho=\sigma / 2 h$ where $h=c / \Omega$ is the ring's vertical halfthickness. Note, though, that this acceleration depends on a radial gradient, so this formula will be problematic at the edge of a sharp-edged ring. However, that problem is avoided by first considering the linear momentum flux $G_{p}$ that pressure effects also transmit through the ring.

The linear momentum flux $G_{p}$ is defined as the force-perlength (e.g., the one-dimensional pressure) that one streamline exerts on that exterior to it due to pressure effects. Thus $\delta f=G_{p} \delta \ell$ is the radial force that one streamline exerts on a neighboring streamline segment whose tangential length is $\delta \ell$. If those streamlines reside in the ring's interior (rather than at its edge), then the net force on a segment is $\Delta f=$ $\delta f(r-\delta r)-\delta f(r) \simeq-(\partial \delta f / \partial r) \delta r=-\left(\partial G_{p} / \partial r\right) \delta r \delta \ell$ where $\delta r$ is the segment's radial width. And since $\Delta f=a_{p} \delta m$ where $\delta m=\lambda \delta l=\sigma \delta r \delta \ell$, then the acceleration due to pressure is related to the linear momentum flux via

$$
a_{p}=-\frac{1}{\sigma} \frac{\partial G_{p}}{\partial r}=-\frac{1}{\sigma_{0}} \frac{\partial G_{p}}{\partial a},
$$

noting that $\sigma=\sigma_{0} / J$ and that $\partial r=J \partial a$. A comparison of Equations (51) and (52) shows that the ring's linear momentum flux due to pressure is

$$
G_{p}=c^{2} \sigma=\frac{c^{2} \sigma_{0}}{J},
$$

assuming that $c$ is constant.

Next, Fourier-expand the longitude-dependent $J^{-1}$ factor in Equation (53), which is Equation (48) with $\delta \rightarrow q$ and $\phi \rightarrow m\left(\theta-\theta_{s}-\tilde{\omega}\right)+\eta$ where $\eta$ is from Equation (42b), so

$$
G_{p} \simeq \frac{c^{2} \sigma_{0}}{\sqrt{1-q^{2}}}\left[1+\frac{2\left(1-\sqrt{1-q^{2}}\right)}{q} e^{\mathrm{i}\left[m\left(\theta-\theta_{s}-\tilde{\omega}\right)+\eta\right]}\right]
$$

upon reverting to complex notation. Inserting this into Equation (52) shows that the acceleration due to pressure now has the desired form of Equation (7) where

$$
\begin{gathered}
A_{p r}^{0}=-\frac{c^{2}}{\sigma_{0}} \frac{\partial}{\partial a}\left(\frac{\sigma_{0}}{\sqrt{1-q^{2}}}\right) \\
A_{p r}^{m}=-\frac{2 c^{2}}{\sigma_{0}} \frac{\partial}{\partial a}\left[\frac{\sigma_{0}\left(1-\sqrt{1-q^{2}}\right)}{q \sqrt{1-q^{2}}} e^{\mathrm{i}(\eta-m \tilde{\omega})}\right],
\end{gathered}
$$

with the $r$ subscript indicating that these are radial accelerations. The dimensionless versions of these accelerations are

$$
\alpha_{p r}^{0}(x)=\frac{A_{p r}^{0}}{|a \mathcal{D}|}=-\frac{c^{\prime 2}}{3(m-\epsilon) s} \frac{\partial}{\partial x}\left(\frac{s}{\sqrt{1-q^{2}}}\right)
$$

and $\quad \alpha_{p r}^{m}(x)=\frac{A_{p r}^{m}}{|a \mathcal{D}|}=\bar{\alpha}_{p r}^{m} e^{-\mathrm{i} m \tilde{\omega}}$

where $\quad \bar{\alpha}_{p r}^{m}(x)=-\frac{2 c^{\prime 2}}{3(m-\epsilon) s}\left(\frac{\partial g_{p}}{\partial x}+\mathrm{i} k a g_{p}\right)$

$$
\text { and } \quad g_{p}(x)=\frac{s\left(1-\sqrt{1-q^{2}}\right)}{q \sqrt{1-q^{2}}} e^{\mathrm{i} \eta},
$$

with $c^{\prime}=c / a \Omega=h / a$ being the particles' dispersion velocity in units of their orbital velocity. The $\bar{\alpha}_{p r}^{m}$ quantity is introduced here for convenience, since it is a real quantity when the ring is nearly peri- or apo-aligned. Since $|m \tilde{\omega}| \sim 0$ or $180^{\circ}$ in that case, $q e^{\mathrm{i} \eta} \simeq e^{\prime}$ in Equation (56d), while the terms proportional to $\mathrm{kag}_{p}$ terms are negligible. Most (but not all) of the ring scenarios considered here are in this limit.

Keep in mind that the accelerations in Equations (55) and (56) are only valid for a ring particle that orbits in the ring's interior. Those equations do not apply to particles inhabiting the streamline at the ring's outer edge, since the pressure there is exerted only by the streamline that is orbiting just interior to it. In that case, $\delta f=G_{p} \delta \ell=\delta m a_{p}=\lambda a_{p} \delta \ell$ is the force on a streamline segment having a tangential length $\delta \ell$ due to pressure, so the acceleration on the ring particles there is $a_{p}=G_{p} / \lambda=G_{p} / \sigma_{0} \Delta a$ where $\Delta a$ is the outermost streamline's semimajor axis width. Inserting Equation (54) into $a_{p}$ then provides the coefficients for the acceleration due to pressure at the ring edge, whose dimensionless forms are

$$
\begin{gathered}
\alpha_{p r}^{0}(x)=\frac{A_{p r}^{0}}{|a \mathcal{D}|}=\frac{c^{\prime 2}(a / \Delta a)}{3(m-\epsilon) \sqrt{1-q^{2}}} \\
\text { and } \quad \alpha_{p r}^{m}(x)=\frac{A_{p r}^{m}}{|a \mathcal{D}|}=\bar{\alpha}_{p r}^{m} e^{-\mathrm{i} m \tilde{\omega}} \\
\bar{\alpha}_{p r}^{m}(x)=\frac{2 c^{\prime 2}\left(1-\sqrt{1-q^{2}}\right)}{3(m-\epsilon) q \sqrt{1-q^{2}}}\left(\frac{a}{\Delta a}\right) e^{\mathrm{i} \eta}
\end{gathered}
$$

This derivation also illustrates the utility of deriving the acceleration due to pressure from the linear momentum flux $G_{p}$, since this approach easily handles the discontinuous pressure drop that will occur are the ring's outer edge.

\subsubsection{Ring Viscosity}

The viscous acceleration $\mathbf{a}_{v}$ that a parcel of ring material experiences along one of the Cartesian axes will be denoted as $a_{v i}$, where the $i=1,2,3$ subscript indicates one of the $x, y, z$ axes. That acceleration is

$$
\begin{aligned}
a_{v i}= & \frac{1}{\rho}\left\{\sum_{j=1}^{3} \frac{\partial}{\partial x_{j}}\left[\eta\left(\frac{\partial u_{i}}{\partial x_{j}}+\frac{\partial u_{j}}{\partial x_{i}}-\frac{2}{3} \delta_{i j} \sum_{k=1}^{3} \frac{\partial u_{k}}{\partial x_{k}}\right)\right]\right. \\
& \left.+\frac{\partial}{\partial x_{i}} \zeta \sum_{k=1}^{3}\left(\frac{\partial u_{k}}{\partial x_{k}}\right)\right\},
\end{aligned}
$$


where $\rho$ is the ring's volume density, $\eta$ its shear viscosity, ${ }^{4} \zeta$ is its bulk viscosity, and $u_{i}$ is the velocity along the $i$ th axis (Landau \& Lifshitz 1987). To transform this into cylindrical coordinates, replace $x_{1} \rightarrow r$ so that the radial velocity $u_{1} \rightarrow v_{r}=\dot{r}$ and the tangential velocity $u_{2} \rightarrow v_{\theta}=r \dot{\theta}$ in the above, while the differentials $\partial x_{1} \rightarrow \partial r$ and $\partial x_{2} \rightarrow r \partial \theta$. Next, note that the ring's various quantities, such as its density or noncircular velocity, are all expected to vary rapidly in the radial $j=1=k$ direction, but vary slowly in the tangential direction. Consequently, only the $j=1=k$ terms need to be preserved in the above, so the components of the viscous acceleration simplify to

$$
\begin{gathered}
\rho a_{v r} \simeq \frac{\partial}{\partial r}\left[\left(\frac{4}{3} \eta+\zeta\right) \frac{\partial v_{r}}{\partial r}\right] \\
\rho a_{v \theta} \simeq \frac{\partial}{\partial r}\left(\eta \frac{\partial v_{\theta}}{\partial r}\right) .
\end{gathered}
$$

Note that Equation (59b) implies that $a_{\nu \theta}$ can be $\neq 0$ in a rigidly rotating disk having $v_{r}=r \dot{\theta}$ where $\dot{\theta}$ is constant. But this is unphysical, because it implies that a rigid rotator would also experience a viscous transport of angular momentum. However, this problem is easily fixed by replacing the $\partial v_{\theta} / \partial r$ in Equation (59b) with $r \partial \dot{\theta} / \partial r$, which provides a more physical expression that is also in agreement with other treatments of viscous astrophysical disks (e.g., Lynden-Bell \& Pringle 1974). Also replace the viscosities in the above with $\eta=v_{s} \rho$ and $\zeta=v_{b} \rho$, where $v_{s}$ is the kinematic shear viscosity and $v_{b}$ the kinematic bulk viscosity, and integrate the repaired version of Equations (59) along the vertical direction so that $\int \rho d z \rightarrow \sigma$, which results in

$$
\begin{gathered}
a_{v r} \simeq \frac{1}{\sigma} \frac{\partial}{\partial r}\left[\left(\frac{4}{3} v_{s}+v_{b}\right) \sigma \frac{\partial v_{r}}{\partial r}\right] \\
a_{\nu \theta} \simeq \frac{1}{\sigma} \frac{\partial}{\partial r}\left(v_{s} \sigma r \frac{\partial \dot{\theta}}{\partial r}\right) .
\end{gathered}
$$

Note that the viscous acceleration also depends on radial gradients, which would be problematic at a ring edge. But that difficulty is again avoided by considering the ring's radial flux of angular and linear momentum.

\subsubsection{Angular Momentum Flux}

The viscous angular momentum flux $F_{\nu}(r, \theta)$ is the rate per unit length that one streamline transmits angular momentum to that orbiting just exterior to it via the ring's viscous friction. Thus, $\delta t=F_{v} \delta \ell$ is the torque that a segment of length $\delta \ell$ exerts on its exterior neighbor. If that segment orbits in the ring's interior, then it is also torqued by ring material orbiting just interior to it, so the net torque on that segment is $\Delta t=\delta t(r-\delta r)-\delta t(r) \simeq-(\partial \delta t / \partial r) \delta r=-\left(\partial F_{v} / \partial r\right) \delta \ell \delta r$ where $\delta r$ is the radial spacings between the adjacent streamlines. And since the net torque on this streamline segment is also $\Delta t=r a_{v \theta} \delta m$ where $\delta m=\lambda \delta \ell=\sigma \delta r \delta \ell$ is the segment's mass, this provides a relation between the viscous angular momentum flux $F_{v}$ and the tangential acceleration $a_{\nu \theta}$,

$$
a_{\nu \theta}=-\frac{1}{r \sigma} \frac{\partial F_{\nu}}{\partial r}=-\frac{1}{a \sigma_{0}} \frac{\partial F_{\nu}}{\partial a} .
$$

\footnotetext{
4 Note that the shear viscosity $\eta$ that is used only in Section 2.4.6 is distinct
} from the angle $\eta$ of Equation (42b) that appears elsewhere in this paper.
Comparing this to Equation (60b) shows that the viscous angular momentum flux is

$$
F_{\nu} \simeq-v_{s} \sigma r^{2} \frac{\partial \dot{\theta}}{\partial r} \simeq-\frac{v_{s} \sigma_{0} a^{2}}{J^{2}} \frac{\partial \dot{\theta}}{\partial a} .
$$

The ring's angular velocity is $\dot{\theta}=\Omega+\dot{\theta}_{1} \simeq \Omega[1+2 e \exp (\mathrm{i} \phi)]$ where $\phi=m\left(\theta-\theta_{s}-\tilde{\omega}\right)$ (see Equation (38)), so $\partial \dot{\theta} / \partial a \simeq$ $\partial \Omega / \partial a+2(\Omega / a)(1-J)$ since

$$
1-J=\frac{\partial}{\partial a}\left(e a e^{\mathrm{i} \phi}\right) \simeq a \frac{\partial}{\partial a}[e \exp (\mathrm{i} \phi)]
$$

by Equation (43) when small terms of $\mathcal{O}(e)$ are ignored. The derivative of Equation (18a) provides $\partial \Omega / \partial a \simeq-(3 \Omega / 2 a)[1+$ $\left.(m-\epsilon) \partial \alpha_{r}^{0} / \partial x\right]$ where $\alpha_{r}^{0}=A_{r}^{0} /|a \mathcal{D}|=\alpha_{g r}^{0}+\alpha_{p r}^{0}$ is the axisymmetric part of the ring's gravity + pressure, so $\partial \dot{\theta} / \partial a \simeq$ $-(3 \Omega / 2 a)\left[4 J / 3-1 / 3+(m-\epsilon) \partial \alpha_{r}^{0} / \partial x\right]$. Inserting this into Equation (62) then yields

$$
F_{\nu} \simeq 2 v_{s} \sigma_{0} a \Omega\left\{\frac{1}{J}-\frac{1}{4 J^{2}}\left[1-3(m-\epsilon) \frac{\partial \alpha_{r}^{0}}{\partial x}\right]\right\}
$$

to lowest order in the streamline's eccentricity $e$. This expression is equivalent to the viscous angular momentum flux that is given in Borderies et al. (1982) when the gradient in the ring's radial acceleration, $\partial \alpha_{r}^{0} / \partial x$, is negligible. Next, Fourierexpand the longitude-dependent factors in $F_{\nu}$, which yields $J^{-2}=[1+2 q \cos (\phi+\eta)] /\left(1-q^{2}\right)^{3 / 2}+$ other terms, with the $J^{-1}$ expansion obtained from Equation (48), so

$$
F_{v} \simeq \frac{3}{2} v_{s} \sigma_{0} a \Omega\left[f_{v}+2 q\left(f_{v}-\frac{4 / 3}{1+\sqrt{1-q^{2}}}\right) e^{\mathrm{i}(\phi+\eta)}\right]
$$

where $f_{v}$ is shorthand for

$$
f_{\nu}(x)=\left(1-q^{2}\right)^{-3 / 2}\left[1+(m-\epsilon) \frac{\partial \alpha_{r}^{0}}{\partial x}-\frac{4}{3} q^{2}\right],
$$

and remembering to preserve only the real part of Equation (65).

The angular momentum luminosity through the ring is the integral about a streamline,

$$
\mathcal{L}_{\nu}(x)=\oint F_{\nu} d \ell=3 \pi f_{\nu} v_{s} \sigma_{0} a^{2} \Omega .
$$

This is equivalent to that given in Borderies et al. (1982) when $\partial \alpha_{r}^{0} / \partial x=0$, which is probably true for most planetary rings. Note that $\mathcal{L}_{v} \propto f_{v}(x)$, and that $f_{v}<0$ when the nonlinearity parameter $q>q^{\star}$ where $q^{\star}=\sqrt{3} / 2 \simeq 0.866$ (again, provided that $\partial \alpha_{r}^{0} / \partial x \simeq 0$; see Equation (66)). This is the threshold for the angular momentum flux reversal that was first described in Borderies et al. (1982). When streamlines become so disturbed that $q>q^{\star}$, the viscous torque causes the streamline's angular momentum to flow inward $\left(\mathcal{L}_{v}<0\right)$ rather than in the usual outward direction. The ring particles orbiting in this disturbed region then spiral inward due to this angular momentum loss, which also opens a gap in the ring.

\subsubsection{Linear Momentum Flux}

The radial component of the ring's viscous acceleration $a_{v r}$ also transmits a flux of linear momentum $G_{v}$ where

$$
a_{v r}=-\frac{1}{\sigma} \frac{\partial G_{v}}{\partial r}=-\frac{1}{\sigma_{0}} \frac{\partial G_{v}}{\partial a}
$$


(see Section 2.4.5). Comparison with Equation (60a) shows that the ring's viscous linear momentum flux is

$$
G_{v}=-\left(\frac{4}{3} v_{s}+v_{b}\right) \sigma \frac{\partial v_{r}}{\partial r}=-\left(\frac{4}{3} v_{s}+v_{b}\right) \frac{\sigma_{0}}{J^{2}} \frac{\partial v_{r}}{\partial a} .
$$

The time derivative of Equation (33) provides the streamline's radial velocity, which is $v_{r}=\dot{r}_{1}=-\mathrm{i} \omega_{m} R_{m} e^{\mathrm{i} \phi} \simeq-\mathrm{i} \epsilon \Omega e a e^{\mathrm{i} \phi}$, so $\partial v_{r} / \partial a \simeq-\mathrm{i} \epsilon \Omega(1-J)=-\mathrm{i} \epsilon \Omega q e^{\mathrm{i}(\phi+\eta)}$ by Equations (43) and (63). Inserting the real part into Equation (69) then yields $G_{v}=-\epsilon\left(\frac{4}{3} v_{s}+v_{b}\right) \Omega \sigma_{0} q \sin (\phi+\eta) / J^{2}$. The Fourier expansion of the longitude-dependent factor in $G_{v}$ is $\sin (\phi+$ $\eta) /[1-q \cos (\phi+\eta)]^{2}=2 \sin (\phi+\eta) /\left[\sqrt{1-q^{2}}\left(1+\sqrt{1-q^{2}}\right)\right]$ + other unimportant terms, so the viscous flux of linear momentum is

$$
G_{v} \simeq \frac{2 \mathrm{i} \epsilon\left(\frac{4}{3} v_{s}+v_{b}\right) \Omega \sigma_{0} q e^{\mathrm{i}(\phi+\eta)}}{\sqrt{1-q^{2}}\left(1+\sqrt{1-q^{2}}\right)}
$$

upon switching back to the complex notation.

\subsubsection{Acceleration Due to Viscosity}

Inserting the linear and angular momentum fluxes $G_{v}$ and $F_{v}$ into Equations (61) and (68) then provides the radial and tangential acceleration that are due to the ring's viscosity, which also have the same form as Equations (7) with

$$
\begin{gathered}
A_{v \theta}^{0}(a) \simeq-\frac{3 \Omega v_{s}}{2 a \sigma_{0}} \frac{\partial}{\partial x}\left(\sigma_{0} f_{\nu}\right) \\
A_{v \theta}^{m}(a) \simeq-\frac{3 \Omega v_{s}}{a \sigma_{0}} \frac{\partial}{\partial x}\left[\sigma_{0} q\left(f_{v}-\frac{4 / 3}{1+\sqrt{1-q^{2}}}\right) e^{\mathrm{i}(\eta-m \tilde{\omega})}\right] \\
\text { and } A_{v r}^{m}(a) \simeq-\frac{2 \mathrm{i} \epsilon \Omega}{a \sigma_{0}}\left(\frac{4}{3} v_{s}+v_{b}\right) \frac{\partial}{\partial x} \\
\\
{\left[\frac{\sigma_{0} q e^{\mathrm{i}(\eta-m \tilde{\omega})}}{\sqrt{1-q^{2}}\left(1+\sqrt{1-q^{2}}\right)}\right], \quad(71 \mathrm{c})}
\end{gathered}
$$

where it is assumed that only $\sigma_{0}, e$, and $\tilde{\omega}$ might vary rapidly with distance $x$ while the $v$ are treated as constants. The dimensionless versions of the tangential accelerations are

$$
\begin{aligned}
& \alpha_{\nu \theta}^{0}(x)=\frac{A_{\nu \theta}^{0}}{|a \mathcal{D}|}=-\frac{v_{s}^{\prime}}{2(m-\epsilon) s} \frac{\partial\left(s f_{\nu}\right)}{\partial x} \\
& \text { and } \quad \alpha_{\nu \theta}^{m}(x)=\frac{A_{\nu \theta}^{m}}{|a \mathcal{D}|}=\bar{\alpha}_{\nu \theta}^{m} e^{-i m \tilde{\omega}},
\end{aligned}
$$

where

$$
\bar{\alpha}_{v \theta}^{m}(x)=-\frac{v_{s}^{\prime}}{(m-\epsilon) s}\left(\frac{\partial g_{\theta}}{\partial x}+\mathrm{i} k a g_{\theta}\right)
$$

with

$$
g_{\theta}(x) \equiv s q\left(f_{v}-\frac{4 / 3}{1+\sqrt{1-q^{2}}}\right) e^{\mathrm{i} \eta}
$$

where $v_{s}^{\prime}=v_{s} / a^{2} \Omega$ is a dimensionless version of the ring's shear viscosity, and $s(x)=\sigma(x) / \sigma_{\infty}$ its fractional surface density. Similarly, the dimensionless version of the radial acceleration is

$$
\text { and }
$$

$$
\alpha_{v r}^{m}(x)=\frac{A_{v r}^{m}}{|a \mathcal{D}|}=\mathrm{i} \bar{\alpha}_{v r}^{m} e^{-\mathrm{i} m \tilde{\omega}}
$$

$$
\text { where } \bar{\alpha}_{v r}^{m}(x)=-\frac{2 \epsilon\left(\frac{4}{3} v_{s}^{\prime}+v_{b}^{\prime}\right)}{3(m-\epsilon) s}\left(\frac{\partial g_{r}}{\partial x}+\mathrm{i} k a g_{r}\right)
$$

$$
\text { with } \quad g_{r}(x) \equiv \frac{s q e^{\mathrm{i} \eta}}{\sqrt{1-q^{2}}\left(1+\sqrt{1-q^{2}}\right)},
$$

where $v_{b}^{\prime}=v_{b} / a^{2} \Omega$. Also note that the $\bar{\alpha}_{v \theta}^{m}$ and $\bar{\alpha}_{v r}^{m}$ become real quantities when the ring is peri- or apo-aligned.

Again, these accelerations are only valid for ring particles orbiting in the ring's interior, and do not apply to particles that inhabit the streamline at the ring's outer edge. In that case, $\delta t=F_{\nu} \delta l=\delta m r a_{v}=\lambda r a_{\nu} \delta l$ is the viscous torque that is exerted on a streamline segment on the edge that has a tangential length $\delta \ell$, so the viscous acceleration on the ring particles there is $a_{v}=F_{v} / \lambda r \simeq F_{v} / \sigma_{0} a \Delta a$ where $\Delta a$ is the outermost streamline's semimajor axis width. Inserting Equation (65) into $a_{v}$ then provides the coefficients for the tangential viscous acceleration,

$$
\begin{aligned}
& \alpha_{\nu \theta}^{0}(x)=\frac{A_{\nu \theta}^{0}}{|a \mathcal{D}|}=\frac{\nu_{s}^{\prime} f_{\nu}}{2(m-\epsilon)}\left(\frac{a}{\Delta a}\right) \\
& \text { and } \quad \alpha_{\nu \theta}^{m}(x)=\frac{A_{\nu \theta}^{m}}{|a \mathcal{D}|}=\bar{\alpha}_{\nu \theta}^{m} e^{-i m \tilde{\omega}},
\end{aligned}
$$

$$
\text { where } \bar{\alpha}_{\nu \theta}^{m}(x)=\frac{v_{s}^{\prime} q e^{i \eta}}{m-\epsilon}\left(f_{v}-\frac{4 / 3}{1+\sqrt{1-q^{2}}}\right)\left(\frac{a}{\Delta a}\right) \text {, }
$$

for particles orbiting in the ring's outermost streamline. Similarly, the radial component of the viscous acceleration is $a_{v r}=G_{v} / \sigma_{0} \Delta a$ for particles in the outermost streamline, so the dimensionless version of this acceleration is

$$
\alpha_{v r}^{m}(x)=\frac{A_{v r}^{m}}{|a \mathcal{D}|}=i \bar{\alpha}_{v r}^{m} e^{-\mathrm{i} m \tilde{\omega}}
$$

$$
\text { where } \bar{\alpha}_{v r}^{m}(x)=\frac{2 \epsilon\left(\frac{4}{3} v_{s}^{\prime}+v_{b}^{\prime}\right) q e^{\mathrm{i} \eta}}{3(m-\epsilon) \sqrt{1-q^{2}}\left(1+\sqrt{1-q^{2}}\right)}\left(\frac{a}{\Delta a}\right) \text {. }
$$

Finally, note that there are several known sources of viscosity in planetary rings: interparticle collisions which have $v \propto \sigma$ in an optically thin ring (Goldreich \& Tremaine 1982), and self-gravitating wakes that result in $v \propto \sigma^{2}$ (Daisaka et al. 2001). $N$-body simulations also indicate that the viscous overstability varies as $v \sim \sigma^{2}$ (see Figure 13 of Salo et al. 2001). Alternatively, one could also have employed a more 
sophisticated viscous stress tensor to account for the effects of collisions (Borderies et al. 1983; Mosqueira \& Estrada 2002). However, previous studies have shown that the surface density near a perturbed ring-edge tends to be nearly constant (Borderies et al. 1989), so the results obtained here are not expected to be particularly sensitive to any viscosity law. In light of this, a simple constant-viscosity law is employed here. Nonetheless, if a power-law $v \propto \sigma^{c}$ viscosity were instead preferred, then the formulae derived here may be adapted.

\subsubsection{Drag}

Small ring particles are also susceptible to drag forces, such as Poynting-Robertson (PR) and plasma drag (Goldreich \& Tremaine 1982), atmospheric drag (Goldreich \& Porco 1987), and possibly the Yarkovsky effect (Rubincam 2006). Drag forces can cause particles to migrate radially, and are particularly effective at damping orbital eccentricities. Although large ring particles are relatively immune to drag forces, they can still experience the effects of drag indirectly by colliding with smaller drag-sensitive ring particles.

Most drag forces vary with the particle's velocity relative to the local circular speed $\Delta \mathbf{v}$, so this work will assume that the acceleration on a ring particle due to drag has the generic form $\mathbf{a}_{d}=-C_{d} \Omega \Delta \mathbf{v}$ where $C_{d}$ is a dimensionless drag coefficient whose value will depend on particulars of the unspecified drag force and the particle size. Since $\Delta \mathbf{v}=$ $\dot{r} \hat{\mathbf{r}}+(r \dot{\theta}-a \Omega) \hat{\theta}=(\hat{\theta}-\mathrm{i} \epsilon \hat{\mathbf{r}}) e a \Omega e^{\mathrm{i} \phi}$ where $\phi=m \theta_{0}+\omega_{m} t-m \tilde{\omega} \simeq$ $m\left(\theta-\theta_{s}-\tilde{\omega}\right)$ (from Equations (33) and (38)), this drag acceleration has the same form as Equations (7) whose radial and tangential components are $A_{d \theta}^{m}=-C_{d} e a \Omega^{2} e^{-\mathrm{i} m \tilde{\omega}}$ and $A_{d r}^{m}=$ $-\mathrm{i} \epsilon A_{d \theta}^{m}$. The dimensionless versions of these drag accelerations are

$$
\alpha_{d r}^{m}(x)=\frac{A_{d r}^{m}}{|a \mathcal{D}|}=\mathrm{i} \bar{\alpha}_{d r}^{m} e^{-\mathrm{i} m \tilde{\omega}}
$$

$$
\text { where } \quad \bar{\alpha}_{d r}^{m}(x)=\frac{\epsilon C_{d} e}{3(m-\epsilon)}
$$

$$
\text { and } \quad \alpha_{d \theta}^{m}(x)=\frac{A_{d \theta}^{m}}{|a \mathcal{D}|}=\bar{\alpha}_{d \theta}^{m} e^{-\mathrm{i} m \tilde{\omega}}
$$

$$
\text { where } \quad \bar{\alpha}_{d \theta}^{m}(x)=-\frac{C_{d} e}{3(m-\epsilon)} \text {. }
$$

This drag force will damp the ring particle's eccentricity at a rate that may be obtained by inserting the drag accelerations $A_{d r}^{m}$ and $A_{d \theta}^{m}$ into the equation of motion (21), which yields

$$
\frac{d e}{d t}=-\frac{3}{2} C_{d} e \Omega
$$

This corresponds to an $e$-damping timescale of

$$
\tau_{e}=\left|\frac{e}{d e / d t}\right|=\frac{2}{3 C_{d} \Omega}=\frac{P_{\mathrm{orb}}}{3 \pi C_{d}}
$$

where $P_{\text {orb }}=2 \pi / \Omega$ is the particle's orbit period.

\subsection{Dimensionless Equation of Motion}

The equation for the particle's motion is Equation (1) with $\mathbf{a}=\mathbf{a}_{g}+\mathbf{a}_{p}+\mathbf{a}_{v}+\mathbf{a}_{d}$ being the acceleration due to the ring's gravity, pressure, viscosity, and a possible drag. Now that the ring's internal forces are suitably Fourier-decomposed (Equations (49), (55), and (71)-(76)), insert those as well as the anticipated solution for the particle's motion (Equation (33)) into its equation of motion (21) to obtain

$$
D \mathcal{R}_{m}=-\Psi_{c}^{m}=-\Psi_{s}^{m}+\frac{2 \mathrm{i} \Omega}{\omega_{m}} A_{\theta}^{m}-A_{r}^{m},
$$

where $D(r)=\kappa^{2}-\omega_{m}^{2}$ is again the particle's frequencydistance from resonance. The dimensionless version of this equation is obtained by dividing by $|a \mathcal{D}|$ and noting that $\mathcal{R}_{m}=e a \exp (-\mathrm{i} m \tilde{\omega})$ and $\omega_{m}=\epsilon \kappa \simeq \epsilon \Omega$ near a resonance, so

$$
\epsilon e d e^{-\mathrm{i} m \tilde{\omega}}-2 \mathrm{i} \epsilon \alpha_{\theta}^{m}+\alpha_{r}^{m}+\psi_{s}=0
$$

where $d(x)=D / \mathcal{D}$ is the dimensionless version of $D$, and the constant $\psi_{s}$ is the satellite's dimensionless forcing function, Equation (29b). The radial part of the dimensionless acceleration is the sum of the contributions due to ring gravity, pressure, viscosity, and drag, so $\alpha_{r}^{m}=A_{r}^{m} /|a \mathcal{D}|=\left[\bar{\alpha}_{g r}^{m}+\bar{\alpha}_{p r}^{m}+\right.$ $\left.\mathrm{i}\left(\bar{\alpha}_{v r}^{m}+\bar{\alpha}_{d r}^{m}\right)\right] e^{-\mathrm{i} m \tilde{\omega}}$, while the tangential acceleration is $\alpha_{\theta}^{m}=$ $A_{\theta}^{m} /|a \mathcal{D}|=\left(\bar{\alpha}_{v \theta}^{m}+\bar{\alpha}_{d \theta}^{m}\right) e^{-\mathrm{i} m \tilde{\omega}}$ by Equations (50, 56-57, 72-75, and 76). Then let $\bar{\alpha}_{c r}^{m}=\bar{\alpha}_{g r}^{m}+\bar{\alpha}_{p r}^{m}$, which is the sum of all the accelerations that are due to conservative forces (gravity plus pressure) while $\psi_{d}=-\left(\bar{\alpha}_{v r}^{m}+\bar{\alpha}_{d r}^{m}\right)+2 \epsilon\left(\bar{\alpha}_{v \theta}^{m}+\bar{\alpha}_{d \theta}^{m}\right)$ becomes the sum of all the dissipative accelerations (viscosity plus drag), so that the equation of motion becomes

$$
\left(\epsilon d e+\bar{\alpha}_{c r}^{m}-\mathrm{i} \psi_{d}\right) e^{-\mathrm{i} m \tilde{\omega}}+\psi_{s}=0,
$$

where $\psi_{d}$ is called the dissipative forcing function, in analogy with Equation (19). The factor $d(x)=D / \mathcal{D}$ in the above is obtained by inserting Equations (18) into $D$ to show that

$$
d(x)=x-(3 \epsilon-m)\left(\alpha_{g r}^{0}+\alpha_{p r}^{0}\right)
$$

where $x$ is understood to be the fractional distance from the nominal resonance, which is where the resonance would be if the ring's internal accelerations were zero. Equation (82) thus shows how the axisymmetric part of the ring's radial acceleration will displace the resonance.

The equation of motion (81) is complex, so its real and imaginary parts provide two coupled equations for the streamline's forced orbital elements $e(x)$ and $\tilde{\omega}(x)$. However, those equations decouple when the dissipation is weak, $\left|\psi_{d}\right| \ll\left|\psi_{s}\right|$, which also results in a ring that is nearly peri- or apo-aligned such that $|m \tilde{\omega}| \ll 1$. In that case, the accelerations $\bar{\alpha}_{c r}^{m}$ and $\psi_{d}$ are all real and depend only on $e(x)$, so the real and imaginary parts of Equation (81) yield

$$
\epsilon e d+\bar{\alpha}_{c r}^{m}+\psi_{s} \simeq 0
$$

$$
\text { and } \quad \tilde{\omega} \simeq \frac{\psi_{d}}{m \psi_{s}}
$$

As one might expect, a numerical algorithm that uses the approximate decoupled equations of motion (83) will converge to a solution much faster than one that attempts to solve the 
exact coupled Equation (81). But keep in mind that these approximate solutions to the equations of motion are only valid when $|m \tilde{\omega}| \ll 1$ and $|k a e| \ll\left|e^{\prime}\right|$.

Lastly, we note that this problem also has a third unknown, the ring's surface density $\sigma_{0}(a)$ or, equivalently, its fractional surface density $s(x)$. To address this, a third equation for this quantity is derived below, which is obtained by requiring that all of the torques exerted on each streamline balance to zero.

\subsection{Angular Momentum Transport}

Two mechanisms transport angular momentum through the ring: the ring's viscosity, and the satellite's gravitational torque. The rate at which this transport occurs is the ring's angular momentum luminosity, and it has two parts, $\mathcal{L}(a)=\mathcal{L}_{v}+$ $\mathcal{L}_{s}$, where $\mathcal{L}_{v}$ is the viscous angular momentum luminosity (Equation (67)), and $\mathcal{L}_{s}$ is the angular momentum luminosity that is due to the satellite's torque on the ring. Note that $\mathcal{L}$ must also be conserved, i.e., $\partial \mathcal{L} / \partial a=0$, for if this were not the case, then streamlines would gain or lose angular momentum over time, which would also cause them to evolve radially since $\partial \mathcal{L} / \partial a \propto A_{\theta}^{0}$. Consequently, static equilibrium requires $\mathcal{L}(a)$ to be a constant everywhere, which also provides that third equation for the ring's unknown surface density $\sigma_{0}(a)$.

To calculate the ring's angular momentum luminosity $\mathcal{L}_{s}$ that is due to the satellite torque, first consider the specific torque that the satellite exerts on a single ring particle, which is $T_{1}(r, \theta)=$ $\mathbf{r} \times\left(-\nabla \Phi_{s}\right) \cdot \hat{\mathbf{z}}=-\partial \Phi_{s} / \partial \theta=m \phi_{s}^{m}(r) \sin m\left(\theta-\theta_{s}\right)$. The time-averaged torque on the particle is obtained by inserting $r(t)=a+r_{1}$ and $\theta(t)=\theta_{0}+\Omega t+\theta_{1}$ into $T_{1}$, Taylor-expanding to first order in the small quantities $r_{1}$ and $\theta_{1}$ (which are Equations (33) and (37)), and then time-averaging, which yields

$$
\begin{aligned}
T_{1} & \simeq\left\langle m\left(\phi_{s}^{m}+r_{1} \frac{\partial \phi_{s}^{m}}{\partial a}\right)\left[\sin m\left(\theta-\theta_{s}\right)+m \theta_{1} \cos m\left(\theta-\theta_{s}\right)\right]\right\rangle \\
& =\frac{1}{2} m R_{m} \Psi_{s}^{m} \sin (m \tilde{\omega}),
\end{aligned}
$$

where the brackets indicate a time-average over the forcing period $2 \pi /\left|\omega_{m}\right|$. An equivalent expression is also derived in Hahn et al. (1995). The time-averaged torque that the satellite exerts on an entire streamline is then $\delta T_{s}=T_{1} \delta m$, where $\delta m=2 \pi \sigma_{0} a \delta a$ is that streamline's mass and $\delta a$ its radial width. Another useful quantity is the satellite's radial torque density $\partial T_{s} / \partial a=\delta T / \delta a$, so

$$
\frac{\partial T_{s}}{\partial a}=m \pi \sigma_{0} a R_{m} \Psi_{s}^{m} \sin (m \tilde{\omega}) .
$$

Also note that the torque that a streamline exerts on the satellite is simply $-\delta T_{s}$, so the total gravitational torque that is exerted by all streamlines having semimajor axis (sma) interior to $a$ is

$$
\begin{aligned}
T_{r}(a) & =-\oint_{\text {sma }<a} \delta T_{s} \\
& =-\int_{0}^{a} m \pi \sigma_{0} a R_{m}\left(a^{\prime}\right) \Psi_{s}^{m} \sin \left(m \tilde{\omega}\left(a^{\prime}\right)\right) d a^{\prime}
\end{aligned}
$$

This torque is a second-order effect since it is the product of two small quantities - the satellite's forcing $\Psi_{s}^{m}$ and the streamline's epicyclic response $R_{m}$. This explains why the ring-satellite torque was formally absent from the linearized equations of motion (12a). And since Equation (86) is the torque that the ring interior to $a$ exerts on the satellite, it is also the luminosity of angular momentum $\mathcal{L}_{s}$ that the ring material interior to $a$ transmits gravitationally to the satellite, which becomes

$$
\begin{gathered}
\mathcal{L}_{s}(x)=T_{r}=-\epsilon m f_{\epsilon}^{m} \mu_{s} \mu_{d} \gamma(x) M_{p}(a \Omega)^{2}, \\
\text { where } \gamma(x)=\int^{x} s\left(x^{\prime}\right) e\left(x^{\prime}\right) \sin \left(m \tilde{\omega}\left(x^{\prime}\right)\right) d x^{\prime}
\end{gathered}
$$

when $\Psi_{s}^{m}$ is replaced by Equation (26), $\sigma_{0}=s(x) \sigma_{\infty}$, and $R_{m}=e(x) a$.

The ring's total angular momentum luminosity is

$$
\begin{aligned}
\mathcal{L}(x)=\mathcal{L}_{v}+\mathcal{L}_{g}= & 3 f_{v} s v_{s}^{\prime} \mu_{d} M_{p}(a \Omega)^{2} \\
& -\epsilon m f_{\epsilon}^{m} \mu_{s} \mu_{d} \gamma M_{p}(a \Omega)^{2},
\end{aligned}
$$

which is a constant everywhere when the ring is in static equilibrium. At sites far from the resonance, $e$ and $\gamma \rightarrow 0$ while $s$ and $f_{v} \rightarrow 1$, so $\mathcal{L}=3 v_{s}^{\prime} \mu_{d} M_{p}(a \Omega)^{2}$ is the ring's angular momentum luminosity. This is also the rate at which the unmodeled part of the ring, which lies interior to the simulated region, delivers angular momentum to the simulated part of the ring. Inserting this into Equation (88) and dividing by $\mathcal{L}$ then provides a third equation of motion for the third unknown, the ring's fractional surface density $s(x)$ :

$$
\begin{gathered}
\ell_{v}(x)+\ell_{s}(x)=1, \\
\text { where } \ell_{v}(x)=s f_{v} \\
\text { and } \quad \ell_{s}(x)=-\frac{\epsilon m f_{\epsilon}^{m} \mu_{s}}{3 v_{s}^{\prime}} \gamma(x)
\end{gathered}
$$

are the dimensionless angular momentum luminosities due to viscosity and the satellite's perturbations, in units of $\mathcal{L}$. Equation (89a) can also be used to determine the location of the ring's edge, since the edge streamline at $x=x_{\text {edge }}$ is where the viscous torque is counterbalanced by the satellite's torque. Or, equivalently, the edge is where the ring's angular momentum luminosity $\mathcal{L}\left(x_{\text {edge }}\right)$ is entirely due to the satellite's gravitational torque. Consequently, the ring's edge is the streamline that satisfies $\ell_{s}\left(x_{\text {edge }}\right)=1$ and $\ell_{v}\left(x_{\text {edge }}\right)=0$.

Lastly, we note that if the dissipation in the ring is weak, i.e., $|m \tilde{\omega}| \ll 1$, then $\tilde{\omega} \simeq \psi_{d} / m \psi_{s}$ (see Equation (83b)), and the fractional angular momentum luminosity due to satellite perturbations becomes

$$
\ell_{s}(x) \simeq-\frac{m(m-\epsilon)}{v_{s}^{\prime}} \int^{x} s\left(x^{\prime}\right) e\left(x^{\prime}\right) \psi_{d}\left(x^{\prime}\right) d x^{\prime}
$$

where $\psi_{d}$ is the dissipative forcing function. Most of the ring scenarios considered below will be in this limit.

\section{NUMERICAL SIMULATIONS OF A SHARP-EDGED PLANETARY RING}

The streamline model described above provides three equations of motion, the real and imaginary parts of Equation (81) and the torque-balance Equation (89), which are to be solved for the system's three unknowns, the ring's eccentricity $e(x)$, longitude of periapse $\tilde{\omega}(x)$, and the ring's fractional surface density $s(x)$, where $x$ is the radial coordinate in the ring. Note that this is a coupled set of nonlinear integro-differential equations, since 
the ring's gravitational acceleration requires integrating over the unknown $e(x), \tilde{\omega}(x)$, and $s(x)$ (see Equations (50)), while the accelerations due to pressure and viscosity involve derivatives of these quantities (Equations (56), and (72)-(73)). The next subsection describes how these equations are solved numerically, with the streamline model then applied to the outer edge of Saturn's B ring. The following subsections then show how the simulated outcomes vary with the model's physical parameters, which are the ring's unperturbed surface density $\sigma_{\infty}$, the ring particles' dispersion velocity $c$, the ring kinematic shear and bulk viscosities $v_{s}$ and $v_{b}$, and the drag coefficient $C_{d}$.

\subsection{Numerical Method}

To solve Equations (81) and (89), treat the broad planetary ring as if it were composed of $N$ discrete streamlines that are uniformly spaced with semimajor axes $a_{i}=a_{\text {in }}+(i-1) \Delta a$, where the ring index $i$ ranges between 1 and $N$, and $\Delta a$ is the rings' width in semimajor axis. The $i$ th streamline's eccentricity is $e_{i}=e\left(a_{i}\right)$, its longitude of periapse $\tilde{\omega}_{i}=\tilde{\omega}\left(a_{i}\right)$, and its fractional surface density $s_{i}=\sigma_{0}\left(a_{i}\right) / \sigma_{\infty}$. This discretization then allows integrals like Equations (50) to be replaced with sums over the $e_{i}, \tilde{\omega}_{i}$, and $s_{i}$, with finite differences used to calculate the derivatives that appear in the accelerations due to pressure and viscosity. This results in a coupled system of $3 N$ nonlinear equations for the $3 N$ unknown $e_{i}, \tilde{\omega}_{i}$, and $s_{i}$, which are then straightforward to solve for numerically.

For example, the $m=0$ component of the ring's dimensionless gravitational acceleration, Equation (50a), is the sum $\alpha_{g r}^{0}\left(x_{i}\right)=\sum_{j \neq i}^{N} G_{i j}$ where $G_{i j}=2 \mu_{d} s_{j} \Delta a / 3 \pi(m-\epsilon)\left(x_{j}-\right.$ $\left.x_{i}\right) \sqrt{1-\delta_{i j}^{2}}$ is the gravitational acceleration that streamline $j$ exerts on a particle in streamline $i$, where $\mu_{d}$ is the ring's normalized mass (see Section 2.4.3), and with $\delta_{i j}$ calculated via a similarly quantized version of Equation (45). Note that $G_{i i}=0$ here, because in the local approximation, a straight wire-thin streamline exerts no net gravitational force on the particles inhabiting that streamline. A similar strategy is also used to calculate the dimensionless $m \geqslant 1$ part of the ring's gravity, $\bar{\alpha}_{g r}^{m}\left(x_{i}\right)$; see Equation (50b). Also note that the accelerations due to pressure and viscosity, Equations (56), (72), and (73), require derivatives of $e, \tilde{\omega}$, and $s$, which are calculated numerically using a three-point Lagrangian interpolation scheme (Hildebrand 1956). Lastly, keep in mind that Equations (57), (74), and (75) are the accelerations on the $N$ th streamline at the ring's edge that is exerted by the adjacent $N-1$ streamline, so the right-hand sides of those equations are evaluated at $x_{N-1}=\left(a_{N-1}-a_{r}\right) / a_{r}$.

The number of streamlines $N$, as well as their radial widths $\Delta a$, are both chosen so that the streamline model can readily resolve the disturbances that Mimas excites at the outer edges of the $\mathrm{B}$ ring. The following subsections will show that these disturbances are usually confined to the rings' outermost 50 or so $\mathrm{km}$, so most calculations use $N=300$ streamlines that have radial widths of $\Delta a=0.5 \mathrm{~km}$ so that the total radial extent of the simulated region $w=N \Delta a$ is usually about $w=150 \mathrm{~km}$. For most ring models, this width is broad enough to show that the simulated ring's innermost part furthest from the resonance has in fact adopted the single particle solution, Equation (29a). This indicates that the ring's internal forces there are negligible, and that a wider ring need not be simulated. However, the following simulations will also show that the self-gravity in a more massive ring is able to transmit the satellite's disturbance to greater distances inward of the resonance. To account for the greater reach of these ring's internal forces, the streamlines' radial widths are increased to $\Delta a=1$ or $2 \mathrm{~km}$ so that the total radial extent of the simulated region becomes $w=300$ to 600 $\mathrm{km}$ in these more massive planetary rings.

The streamline model developed here, called NLsgvp, is written in IDL, and it solves the coupled set of $3 N$ nonlinear equations for the streamlines' $3 \mathrm{~N}$ orbit elements and surface densities. Although IDL does supply routines for solving coupled systems of nonlinear equations, they are not particularly robust, and sometimes fail to find a satisfactory solution to these equations. So the MPFIT algorithm is instead used here, which is an IDL procedure written by Craig Markwardt that is available at http://cow.physics.wisc.edu/ craigm/idl/fitting.html. MPFIT is a parameter search algorithm, and it was formally designed to search parameter space for the purpose of fitting a parameterized model to a data set. However, the act of seeking solutions to a coupled set of nonlinear equations is conceptually very similar to modeling data, since we wish to find the set of $3 N$ "parameters" $e_{i}, \tilde{\omega}_{i}$, and $s_{i}$, that best satisfy the $3 N$ Equations (81) and (89), whose right-hand sides are "data." MPFIT is quite efficient and very well suited for solving this type of problem.

MPFIT must be initialized by first providing it with a trial solution. That initial guess is formed from the single particle solution, Equation (29a), which is also the solution to the equation of motion (83a) at sites far from the resonance where the conservative part of ring's internal acceleration, $\bar{\alpha}_{c r}^{m}$, is negligible. Note, however, that Equation (29a) diverges at the resonance where $d(x) \simeq x \rightarrow 0$, whereas $e(x)$ is expected to stay finite inside the nonlinear region where $|x|<x_{\mathrm{NL}}$ (see Equation (44)) due to the ring's internal forces. So to qualitatively mimic this effect, replace $x$ in Equation (29a) with $x \rightarrow-\epsilon \sqrt{x^{2}+x_{\mathrm{NL}}^{2}}$ such that

$$
e_{\text {trial }}(x)=\frac{\psi_{s}}{\sqrt{x^{2}+\left|\psi_{s}\right|}},
$$

where $\psi_{s}$ is the satellite's dimensionless forcing function, Equation (29b). This is the trial solution that is used to initialize MPFIT, which also adopts $s(x)=1$ and $\tilde{\omega}(x)=0$.

All of the calculations described below use double-precision arithmetic. To assess the accuracy of the model results, we insert all numerical solutions obtained by NLsgvp back into the equation of motion (81) and divide by $\psi_{s}$, with those solutions also being inserted into the torque-balance equation (89a) subtracted by 1 . The residuals on the right-hand side of those equations are the solutions' fractional errors, which for the models described below are all smaller than $4 \times 10^{-12}$.

\subsection{Simulations of the Outer B Ring}

\subsubsection{Variations with Surface Density}

Figure 1 illustrates how the ring's epicyclic amplitude $R_{m}(x)=a e(x)$ varies with $\sigma_{\infty}$, which is the ring's undisturbed surface density far from the edge. These curves are solutions to Equations (81) and (89), and were obtained using the numerical method described in Section 3.1. The main point of this figure is to show that the epicyclic amplitude $R_{m}$ at the ring edge is larger when the ring has a lower mass. Note that the ring's response approaches the single particle result, Equation (28), as $\sigma_{\infty} \rightarrow 0$. All of these simulations adopt viscosities of $v_{s}=v_{b}=50 \mathrm{~cm}^{2} \mathrm{~s}^{-1}$, which is comparable to the viscosity measured in Saturn's A ring (Tiscareno et al. 2007; Porco et al. 2007). There is no drag in these simulations $\left(C_{d}=0\right)$, and the ring's outer edge is chosen to be at the nominal resonance at 


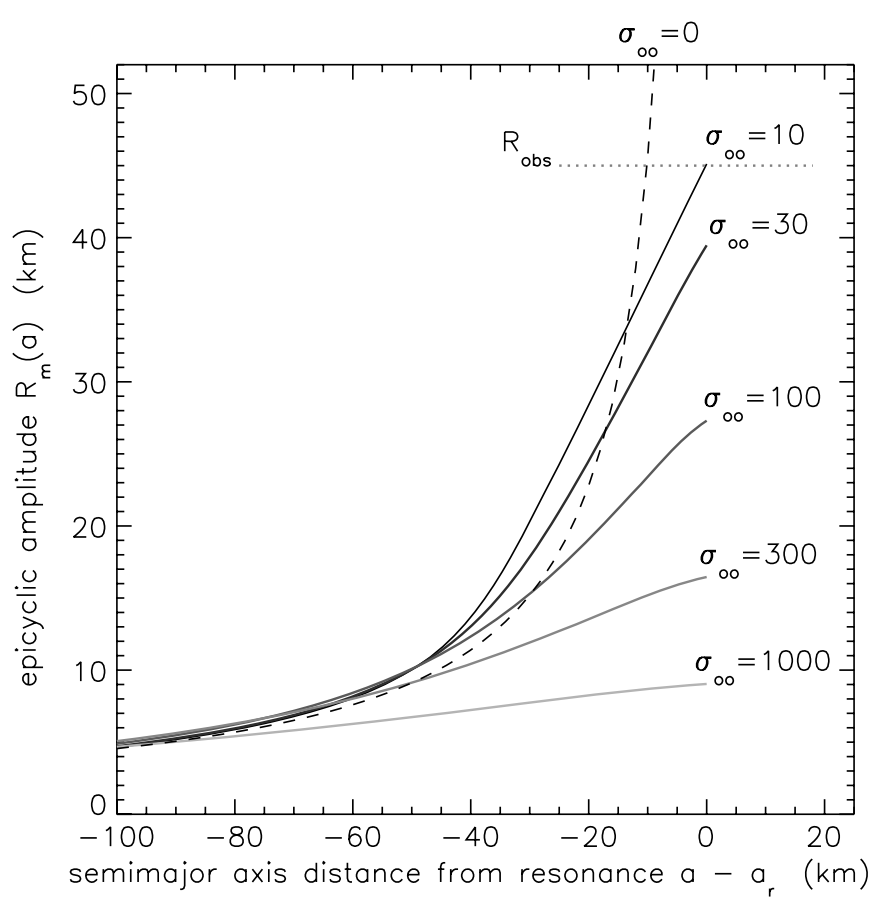

Figure 1. Simulations of the B ring's epicyclic amplitude $R_{m}$ plotted vs. $a-a_{r}$, which is the radial distance from Mimas' nominal $m=2$ ILR. The solid curves are for simulated rings that have a variety of surface densities $\sigma_{\infty}$ that are indicated above in units of $\mathrm{gm} \mathrm{cm}^{-2}$. The dashed $\sigma_{\infty}=0$ curve is the single particle solution, Equation (28), and the horizontal dotted line indicates the B ring's observed epicyclic amplitude of $R_{\mathrm{obs}} \simeq 45 \mathrm{~km}$ (Spitale \& Porco 2006). The model rings all have viscosities of $v_{s}=v_{b}=50 \mathrm{~cm}^{2} \mathrm{~s}^{-1}$, a stability parameter of $Q=2$, and no drag $\left(C_{d}=0\right.$.) The rings' outer edges are also forced to reside at the nominal resonance where $x=0=a-a_{r}$; see Section 3.2.1 for details. All of these simulations use $N=300$ streamlines that are distributed uniformly at the B ring's outer edge. The streamlines' radial widths differ in each model, with $\Delta a=0.50,0.50,0.67,1.0$, and $2.0 \mathrm{~km}$, with wider streamlines used in the heavier rings for reasons explained in Section 3.1. The total radial extent of the modeled region is $w=N \Delta a=150 \mathrm{~km}$ in the lightest ring and $w=600 \mathrm{~km}$ in the heaviest ring. The torque $T_{s}$ that the satellite exerts on the ring is Equation (86) evaluated at the ring's outer edge, and it is quoted in units of $T_{G T}$ (Equation (95)). Those torque ratios range over $\left|T_{s} / T_{G T}\right|=7.6 \times 10^{-4}$ for the lighter $\sigma_{\infty}=10 \mathrm{gm} \mathrm{cm}^{-2}$ ring to $\left|T_{s} / T_{G T}\right|=1.7 \times 10^{-7}$ in the heavier $\sigma_{\infty}=1000 \mathrm{gm} \mathrm{cm}^{-2}$ ring.

$x=0=a-a_{r}$. The ring particles' dispersion velocity $c$ is also chosen such that the ring is marginally gravitationally stable. A gravitationally stable ring has a $Q>1$ (Toomre 1964), where $Q \simeq c \Omega / \pi G \sigma_{\infty}=c^{\prime} / \mu_{d}$. However, Saturn's main A and B rings also exhibit brightness asymmetries that are believed to be due to the presence of self-gravitating wakes, but those wakes only occur when $Q \lesssim 2$ (Salo 1992). Consequently, a higher mass but marginally stable ring also has a larger dispersion velocity, and the simulations reported in Figure 1 have $c$ chosen so that $Q=2$. The dotted horizontal line also indicates the B ring's observed epicyclic amplitude, $R_{\mathrm{obs}} \simeq 45 \mathrm{~km}$, which was measured in Cassini spacecraft observations that were acquired in 2005 (Spitale \& Porco 2006). Figure 1 tentatively suggests that the outer edge of the $\mathrm{B}$ ring might have a surface density as low as $\sigma_{\infty} \sim 10 \mathrm{gm} \mathrm{cm}^{-2}$.

Figure 2 plots the nonlinearity parameter $q$ versus distance from resonance for the simulations described in Figure 1. In all of these simulations, $|k a e| \ll\left|e^{\prime}\right|$, so the nonlinearity parameter $q \simeq\left|e^{\prime}\right|$ is also the ring's eccentricity gradient. Figure 2 shows that the disturbed edges of lighter rings tend to be more nonlinear than heavier rings. The ring's epicyclic amplitude $R_{m}$ is also proportional to the satellite's mass, so increasing the perturber's mass also makes the ring more nonlinear.

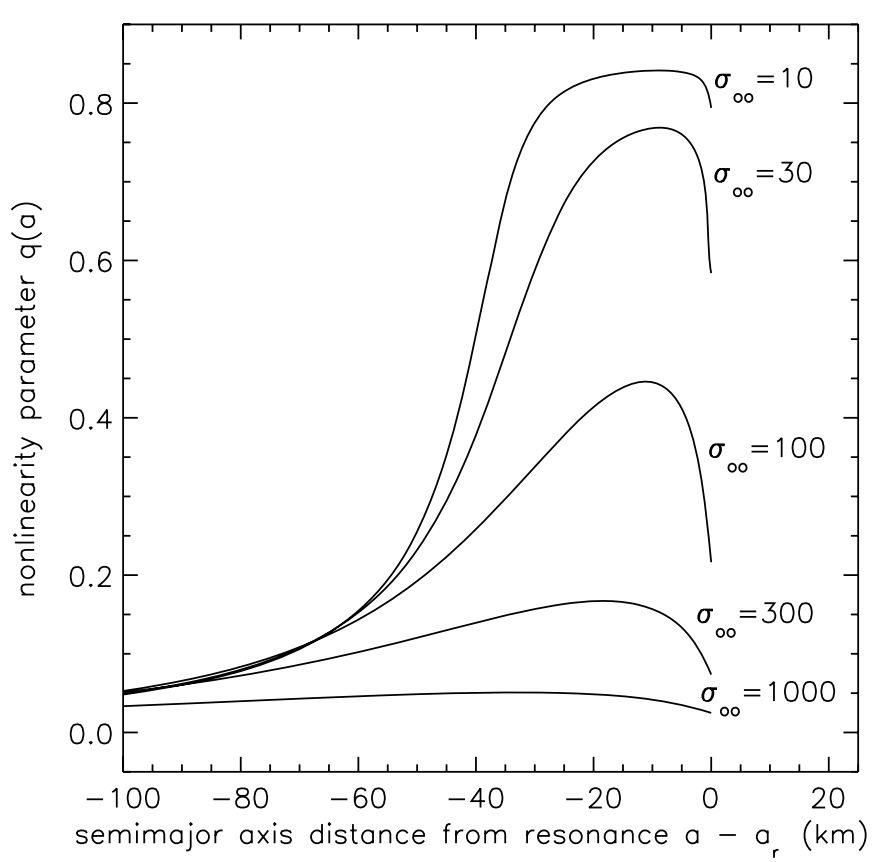

Figure 2. Nonlinear parameter $q$ plotted vs. distance from resonance for the B ring models of Figure 1.

\subsubsection{Variations in the Ring-Edge Location}

Section 2.3 notes that the outer edge of the B ring could lie as far as $24 \mathrm{~km}$ exterior to Mimas' nominal $m=2$ ILR. This displacement was first measured by Porco et al. (1984) from Voyager observations, but with a large uncertainty. However, a preliminary analysis of Cassini observations of the B ring, which is described in Spitale \& Porco (2006), does indicate that the ring-edge lies about 20 or so $\mathrm{km}$ beyond the resonance.

Figure 3 illustrates the consequences of displacing the $\mathrm{B}$ ring's edge radially outward; the three curves show the ring's epicyclic amplitude $R_{m}(a)$ for simulated rings whose outermost semimajor axis lies a distance $\Delta a=0,10$, and $25 \mathrm{~km}$ exterior to the nominal resonance location. Figure 3 also shows that the ring's epicyclic amplitude $R_{m}(a)$ grows linearly as the edge is approached, even in the portion of the ring edge that lies exterior the resonance. Consequently, letting the ring edge lie farther beyond the resonance will result in a larger epicyclic amplitude. However, Section 3.2.1 shows that the increase in the epicyclic amplitude can be offset by increasing the ring's surface density $\sigma_{\infty}$. So Figure 3 assigns a larger surface density to rings whose outer edges are displaced farther outward, but in a manner that keeps the epicyclic amplitude at the edge comparable to the observed value, $R_{\mathrm{obs}} \simeq 45 \mathrm{~km}$, which is dotted line in Figure 3 . The rightmost curve in Figure 3, which agrees with the B ring's observed epicyclic amplitude as well as the likely upper limit on the edge's location, now shows that the B ring could have a much higher surface density of $\sigma_{\infty} \sim 280 \mathrm{gm} \mathrm{cm}^{-2}$.

\subsubsection{Variations with Dispersion Velocity}

Figure 4(a) shows that the ring's epicyclic amplitude $R_{m}$ is quite insensitive to the particles dispersion velocity $c$. Those simulations are for a B ring that has a surface density fixed at $\sigma_{\infty}=30 \mathrm{gm} \mathrm{cm}^{-2}$ and a stability parameter that ranges over $0 \leqslant Q \leqslant 12$, which is a convenient proxy for $c$ since $Q \simeq c \Omega / \pi G \sigma_{\infty}$. These $Q$ values correspond to dispersion velocities of $c \leqslant 4.9 \mathrm{~mm} \mathrm{~s}^{-1}$ and vertical scale heights $h=c / \Omega$ 


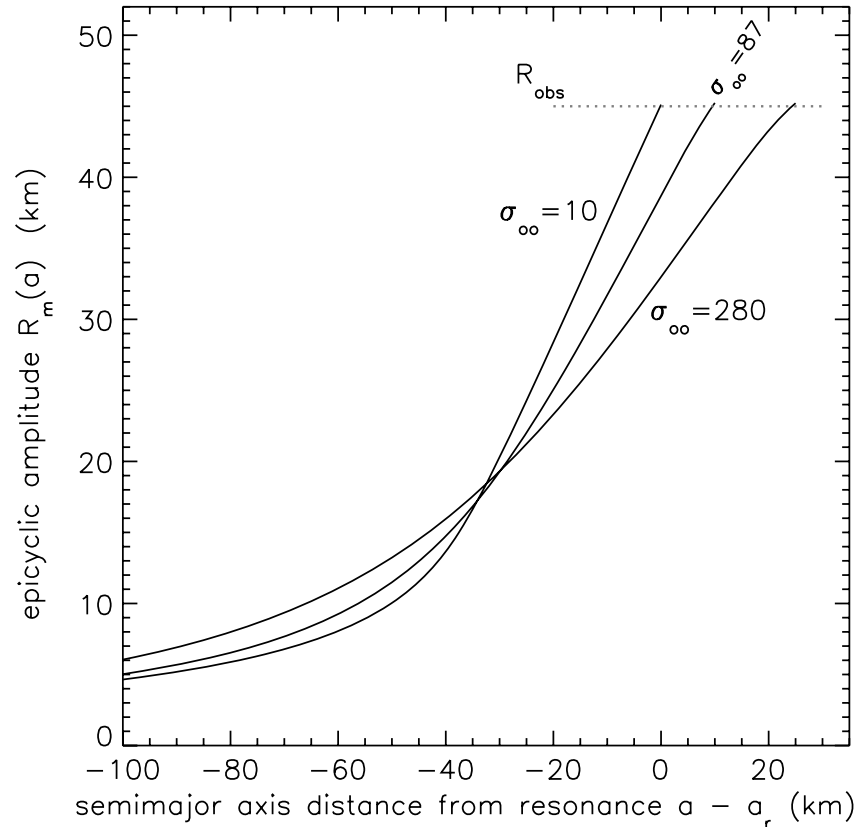

Figure 3. Results of three simulations of the outer B ring as it is perturbed by Mimas' $m=2$ ILR. The three simulated rings have outer edges that lie at radial distances of $a-a_{r}=0,10$, and $25 \mathrm{~km}$ beyond the nominal $m=2$ resonance position. Labels indicate each ring's surface density $\sigma_{\infty}$ in units of $\mathrm{gm} \mathrm{cm}^{-2}$, which is adjusted so that the epicyclic amplitude $R_{m}$ at the ring's outer edge is comparable to the observed value $R_{\mathrm{obs}} \simeq 45 \mathrm{~km}$, which is indicated by the dotted line. These models use $N=300$ streamlines having widths of $\Delta a=0.50,0.87$, and $1.42 \mathrm{~km}$, with wider streamlines being used in the heavier rings, so the total radial extent of the modeled regions are 150, 260, and 425 $\mathrm{km}$. The remaining model parameters are identical to that used in Figures 1-2, with $v_{s}=v_{b}=50 \mathrm{~cm}^{2} \mathrm{~s}^{-1}, Q=2$, and $C_{d}=0$. The torque that the satellite exerts on the ring ranges over $\left|T_{S} / T_{G T}\right|=7.6 \times 10^{-4}$ in the lighter $\sigma_{\infty}=10$ $\mathrm{gm} \mathrm{cm}^{-2}$ ring to $\left|T_{S} / T_{G T}\right|=2.9 \times 10^{-5}$ in the heavier $\sigma_{\infty}=280 \mathrm{gm} \mathrm{cm}^{-2}$ ring.

of $h \leqslant 32 \mathrm{~m}$. Note that the black $Q=0$ curve, which represents a pressureless ring, is indistinct from the other $Q \leqslant 5$ models. Figures 4(b) and (c) plot the simulated rings' fractional surface densities $s(a)$ and longitudes of periapse $\tilde{\omega}(a)$ versus semimajor axis $a$, which is able to distinguish the dynamically hotter $Q \gtrsim 10$ models from the cooler $Q \leqslant 5$ models. Note the large variations in $s(a)$ and $\tilde{\omega}$ that is seen at the outer edges seen of the hotter rings that have $Q \geqslant 10$; those variations are due to the larger pressure drop that occurs across the outermost streamline (e.g., Equations (57)). Those surface density excesses seen at the rings' edges are also reminiscent of those occurring in models of narrow ringlets (e.g., Chiang \& Goldreich 2000; Mosqueira \& Estrada 2002), but the variations seen in Figure 4 are poorly resolved due to the models' radial sampling of $\Delta a=0.5 \mathrm{~km}$. Nonetheless, Figure 4 does show that pressure effects, if important at all, are confined to the ring's outermost $\Delta a \lesssim 0.5 \mathrm{~km}$. Since this study is interested in the perturbed ring's state over a much broader radial scale, $\Delta a \sim 40 \mathrm{~km}$ according to B ring models of Figure 4, the following will for simplicity keep $Q=2$ for most of the ring scenarios that are considered below.

Lastly, note that $\tilde{\omega}(a)>0$ in the hotter $Q=12$ model (Figure 4(c)). Equation (85) indicates that the torque that the satellite exerts on this model ring is positive. But that torque has the wrong sign, since a satellite must exert a negative torque on the ring if it is to be confined interior to an ILR. This inability of the satellite to confine a viscous and dynamically hot ring is another reason why hot ring models need not be considered further. The confinement of the B ring by Mimas' gravitational torque is also considered in greater detail below.

\subsubsection{Ring Viscosity, and the Torque-Balance Problem}

Figure 5 shows how the simulated outcomes depend on the ring's viscosity. These models are similar to the $\mathrm{B}$ ring simulations shown in Figure 4, except that the rings' viscosities take values of $v_{s}=v_{b}=5,50$, and $500 \mathrm{~cm}^{2} \mathrm{~s}^{-1}$ while $Q=2$. Note that the rings' epicyclic amplitudes $R_{m}=e a$ are all insensitive to the viscosity $v_{s}=v_{b}$ (Figure 5(a)), as are the rings' fractional surface densities $s$ (Figure 5(b)), and their angular momentum luminosities $\ell_{v}$ and $\ell_{s}$ (Figure 5(d)). This is due to the ring being nearly peri- or apo-aligned, i.e., $|\tilde{\omega}| \simeq\left|\psi_{d} / m \psi_{s}\right| \ll 1$ (see Equation (83b)) in all of these simulations. Since the dissipative forcing function $\psi_{d}$ is small compared to the other terms in the equation of motion, it has little effect on $e$ (see Equations (81) and (83)). However, Figure 5 (c) demonstrates that the streamlines' longitudes of periapse $\tilde{\omega} \propto \psi_{d}$ are proportional to the ring viscosity, since

$$
\psi_{d}=-\bar{\alpha}_{v r}^{m}+2 \epsilon \bar{\alpha}_{\nu \theta}^{m} \equiv \psi_{\nu} \propto\left(v_{s}+f v_{b}\right),
$$

where the factor $f$ is of the order of 1 (see Equations (72) and (73)).

Figure 5(d) also shows that the ring's angular momentum luminosity $\ell_{s}$, which is due to the satellite's gravitational torque on the ring, is small, i.e., $\left|\ell_{s}\right| \ll 1$. This is very problematic, because Section 2.6 showed that the ring's outer edge should be the site where the viscous torque is counterbalanced by the satellite's torque, which is also where $\ell_{v} \rightarrow 0$ and $\ell_{s} \rightarrow 1$. However, this torque-balance requirement is not satisfied by any of the simulations described in Figures 1-5. Although the simulations described above provide useful illustrations of how a ring's simulated outcome depend on its physical properties $\sigma_{\infty}, v$, and $c$, they are all unphysical since they do not achieve a torque-balance at the ring's outer edge. However, Section 3.2.5 does explore an alternative ring scenario that does in fact achieve the desired torque balance.

But first, a final comment on Figure 5(b), which shows that the simulated ring's surface density has a $\sim 20 \%$ excess in its outermost $\sim 20 \mathrm{~km}$. This is a due the conservation of the ring's angular momentum luminosity $\mathcal{L} \simeq \mathcal{L}_{v} \propto s f_{v}$ (see Equation (88) and note that $\mathcal{L}_{s}$ is negligible in these simulations) where the function $f_{v}$ varies approximately as $1-4 q^{2} / 3$ (see Equation (66)) where the nonlinearity parameter is $q \simeq|d e / d x|$, which is also plotted in Figure 5(d). Because the ring' eccentricity gradient gets large near the resonance, the quantity $f_{v}$ is diminished there, but that must be offset by an increase in the ring's fractional surface density $s=\sigma_{0}(a) / \sigma_{\infty}$ in order to conserve $\mathcal{L} \propto s f_{\nu}$.

\subsubsection{Possible B Ring Solution}

Section 3.2.4 notes that all of the ring simulations considered thus far fail to balance the ring's viscous torque against the satellite's gravitational torque, so the proximity of the B ring's outer edge near Mimas' $m=2$ ILR is not yet accounted for. Equation (87) shows that the satellite's torque on the ring is proportional to $\gamma(x)=\int^{x} s e \sin (m \tilde{\omega}) d x^{\prime}$, which is the product of the streamline's forced eccentricity $e(x)$ and the angle by which that streamline lags behind the satellite's longitude, $\tilde{\omega}(x)$. That lag angle depends on the ring's shear and bulk viscosity via $\sin m \tilde{\omega} \propto \psi_{d} \propto v_{s}+f v_{b}$ where $f \sim \mathcal{O}(1)$ (Section 3.2.4), 

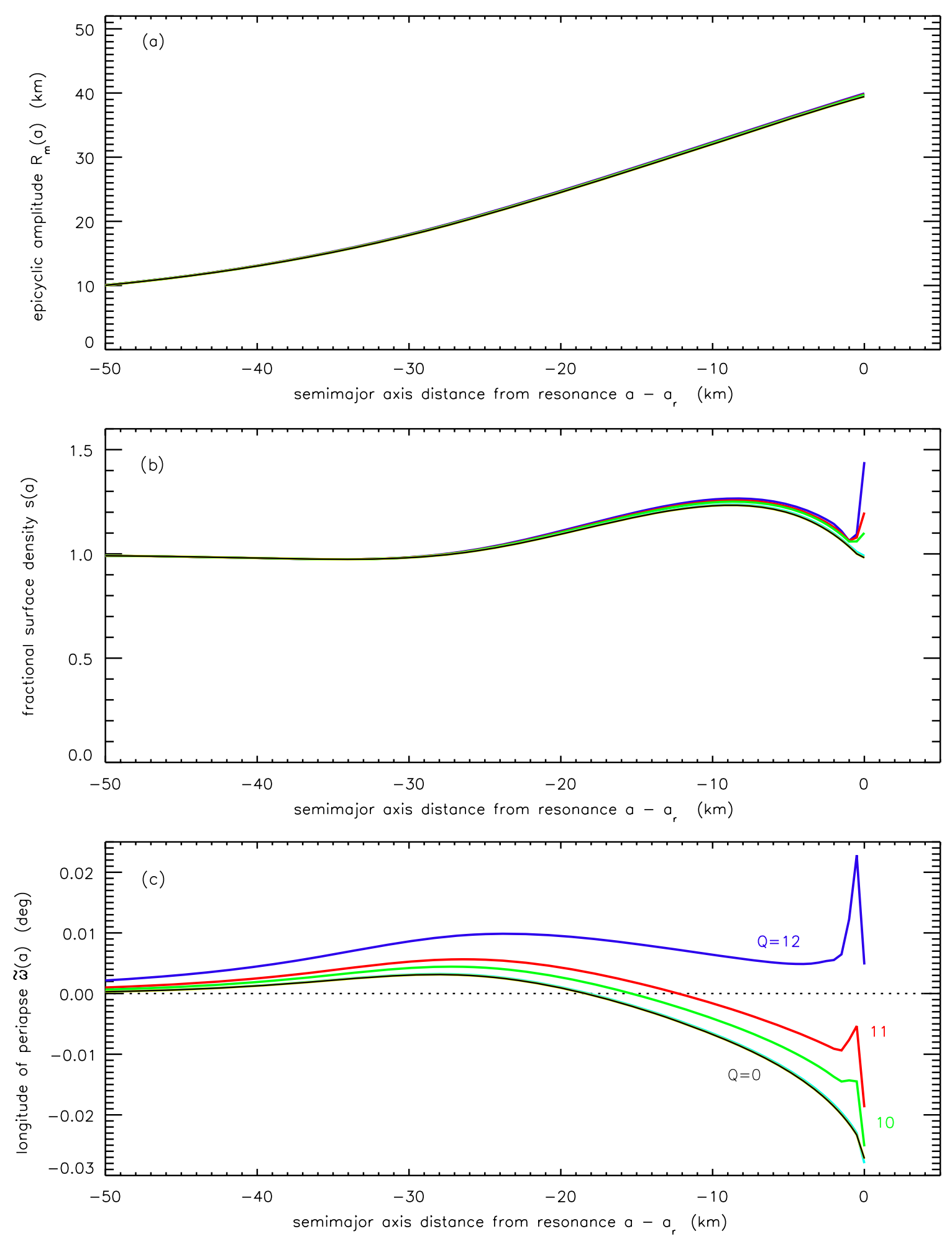

Figure 4. The B ring's epicyclic amplitude $R_{m}(a)$, its fractional surface density $s(a)$, and its longitude of periapse $\tilde{\omega}(a)$ plotted vs. distance from resonance $a-a_{r}$ for models that all have the same surface density $\sigma_{\infty}=30 \mathrm{gm} \mathrm{cm}^{-2}$, viscosities $v_{s}=v_{b}=50 \mathrm{~cm}^{2} \mathrm{~s}^{-1}$, and stability parameters of $Q=0$ (which corresponds to a pressureless ring, black curve), $Q=1$ (yellow), $Q=5$ (cyan), $Q=10$ (green), $Q=11$ (red), and $Q=12$ (blue). The $Q=0-5$ simulations are nearly indistinguishable and lie under the black curves. There is no drag in these simulations $\left(C_{d}=0\right)$, and the rings' outer edges are at the nominal resonance where $a=a_{r}$. These simulations use $N=300$ streamlines that are distributed uniformly over the ring's outermost $150 \mathrm{~km}$, so the radial resolution here is $0.5 \mathrm{~km}$. The torque that the satellite exerts on the ring ranges is $\left|T_{s} / T_{G T}\right|=1.5 \times 10^{-4}$ in the $0<Q \leqslant 5$ models, $7.7 \times 10^{-5}$ in the $Q=10$ model, $6.1 \times 10^{-6}$ for $Q=11$, and $2.3 \times 10^{-4}$ for $Q=12$.

so the satellite's total torque on the ring is controlled by the sum of these viscosities. Although an increase in the ring's shear viscosity $v_{s}$ does increase the satellite's torque, this is of no help here because the viscous torque (Equation (67)) would increase by the same factor, and the torque mismatch would still persist. However, Equation (87) indicates that an increase in the ring's bulk viscosity $v_{b}$ can increase the satellite's torque without altering the viscous torque. 

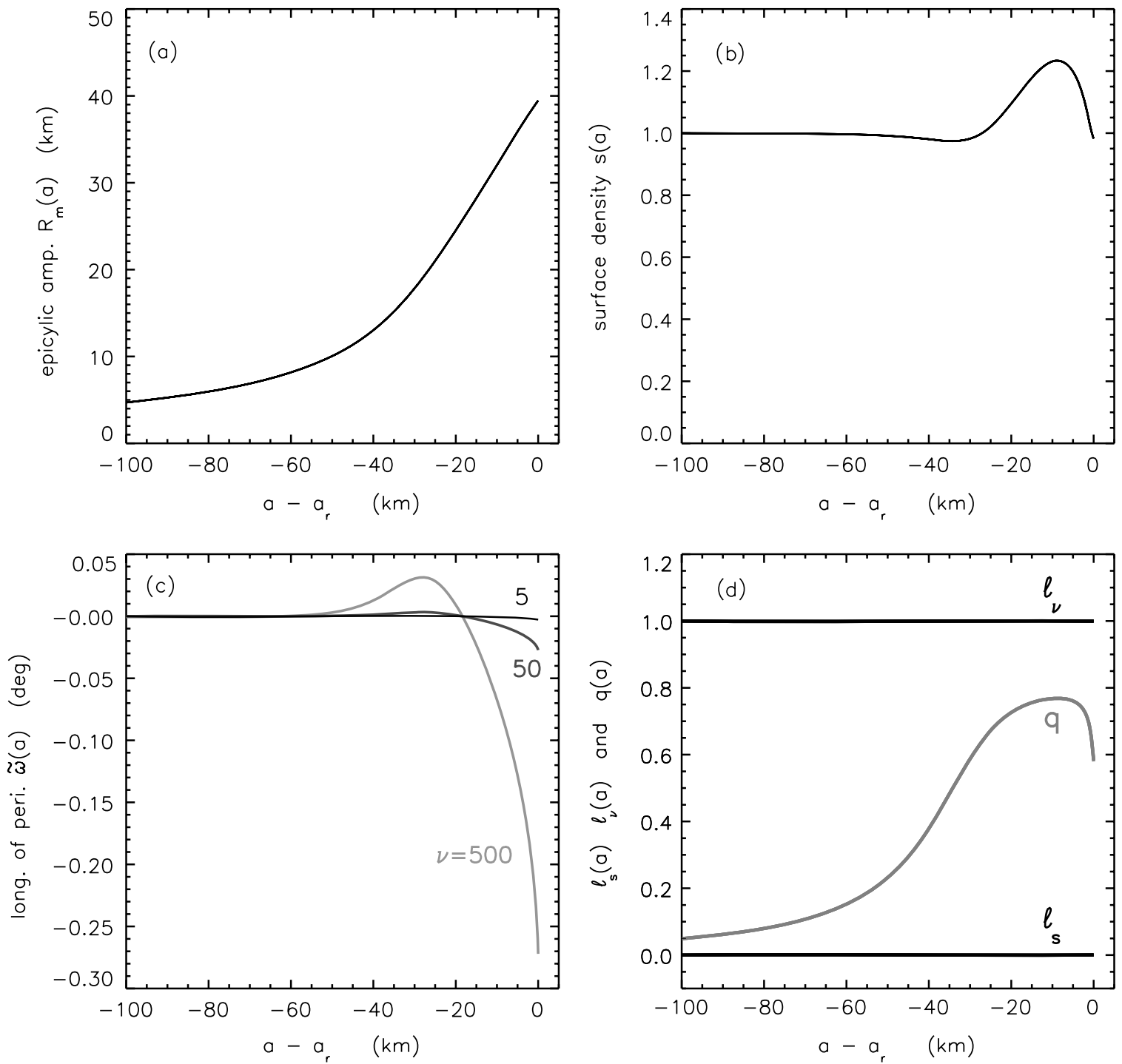

Figure 5. Simulations of the B ring's epicyclic amplitude $R_{m}$ (a), fractional surface density $s$ (b), and longitude of periapse $\tilde{\omega}$ (c) all plotted against the radial distance from resonance $a-a_{r}$. (d) plots the rings' dimensionless angular momentum luminosity due to viscosity $\ell_{v}$ and the satellite's gravitational torque $\ell_{s}$, as well as the rings nonlinearity parameter $q$. Three simulations are shown, and they all have an undisturbed surface density $\sigma_{\infty}=30 \mathrm{gm} \mathrm{cm}^{-2}$, stability parameter $Q=2$ (which corresponds to particle dispersion velocity of $c=0.82 \mathrm{~mm} \mathrm{~s}^{-1}$ and a ring vertical half-thickness of $h=5.4 \mathrm{~m}$ ). The ring's outer edge is also placed at resonance at $a=a_{r}$, and the drag coefficient $C_{d}=0 . N=300$ streamlines are used to model the ring's outermost $w=N \Delta a=150 \mathrm{~km}$ with a spatial sampling of $\Delta a=0.5 \mathrm{~km}$. These three simulations do have distinct viscosities $v_{s}=v_{b}=5,50$, and $500 \mathrm{~cm}^{2} \mathrm{~s}^{-1}$, which are indicated by the labels in (c). Note also that the curves in (a), (b), and (d) all lie on top of each other for each of the three simulations, due to $R_{m}, s, q$, and $\ell$ being insensitive to the choice of $v_{s}=v_{b}$. The torque that the satellite exerts on the ring is $\left|T_{s} / T_{G T}\right|=1.5 \times 10^{-5}, 1.5 \times 10^{-4}$, and $1.5 \times 10^{-3}$ for the $v_{s}=v_{b}=5,50$, and $500 \mathrm{~cm}^{2} \mathrm{~s}^{-1}$ ring models.

But first, a comment on measurements of the viscosity of planetary rings. The shear viscosity $v_{s}$ is, as the name suggests, a measure of the friction that results from the ring's shearing motions. On the other hand, the ring's bulk viscosity $v_{b}$ is the friction that occurs due to the ring's compressive or decompressive motions, which explains why $v_{b}$ enters into the equations of motion when there is a gradient in the ring's radial velocity (Equation (59a)). One way to infer $v_{s}$ and $v_{b}$ is to examine spacecraft observations of spiral density waves; these waves are damped by viscous effects over the radial scale $\Delta r_{v} \propto\left(v_{s}+\frac{3}{7} v_{b}\right)^{-1 / 3}$ (Goldreich \& Tremaine 1978; Shu 1984), so a measurement of $\Delta r_{v}$ provides an estimate of the combined viscosities $v_{s}+\frac{3}{7} v_{b}$. Tiscareno et al. (2007) use Cassini observations of spiral density waves in Saturn's A ring to infer viscosities of $30 \lesssim v \lesssim 300 \mathrm{~cm}^{2} \mathrm{~s}^{-1}$ there. However, Tiscareno et al. (2007) dropped the bulk viscosity $v_{b}$ from their Equation (7), so the viscosities quoted there should instead be interpreted as the combination $v \rightarrow v_{s}+\frac{3}{7} v_{b}$. Similarly, Equations (73) and (75) indicate that our simulations will be sensitive to the combination $v_{s}+\frac{3}{4} v_{b}$. Porco et al. (2007) also use the widths of the Keeler and Encke gaps in Saturn's A ring to infer viscosities of $20 \lesssim v \lesssim 87 \mathrm{~cm}^{2} \mathrm{~s}^{-1}$, but again these viscosities are likely measurements of some combination of $v_{s}$ and $v_{b}$. The upshot is that current ring observations only constrain the sum of $v_{s}$ and $v_{b}$, and that $v_{s}$ and $v_{b}$ are not known individually.

With this in mind, Figure 6 shows the results of three B ring simulations whose viscosities sum to $v_{s}+v_{b}=100 \mathrm{~cm}^{2}$ $\mathrm{s}^{-1}$ (which is roughly the A ring's total viscosity), while their ratios obey $v_{b} / v_{s}=0,1,1000$, and 7873 . That last ratio was chosen so that the viscous and satellite torques do indeed balance at the ring's outer edge, which is where $s \rightarrow 0$ while $\ell_{v} \rightarrow 0$ and $\ell_{s} \rightarrow 1$ (black curves in Figures 6(b) and (d)). These simulations' other parameters are similar to that adopted previously in Figure 5, with $\sigma_{\infty}=30 \mathrm{gm} \mathrm{cm}^{-2}, Q=2, C_{d}=0$, 

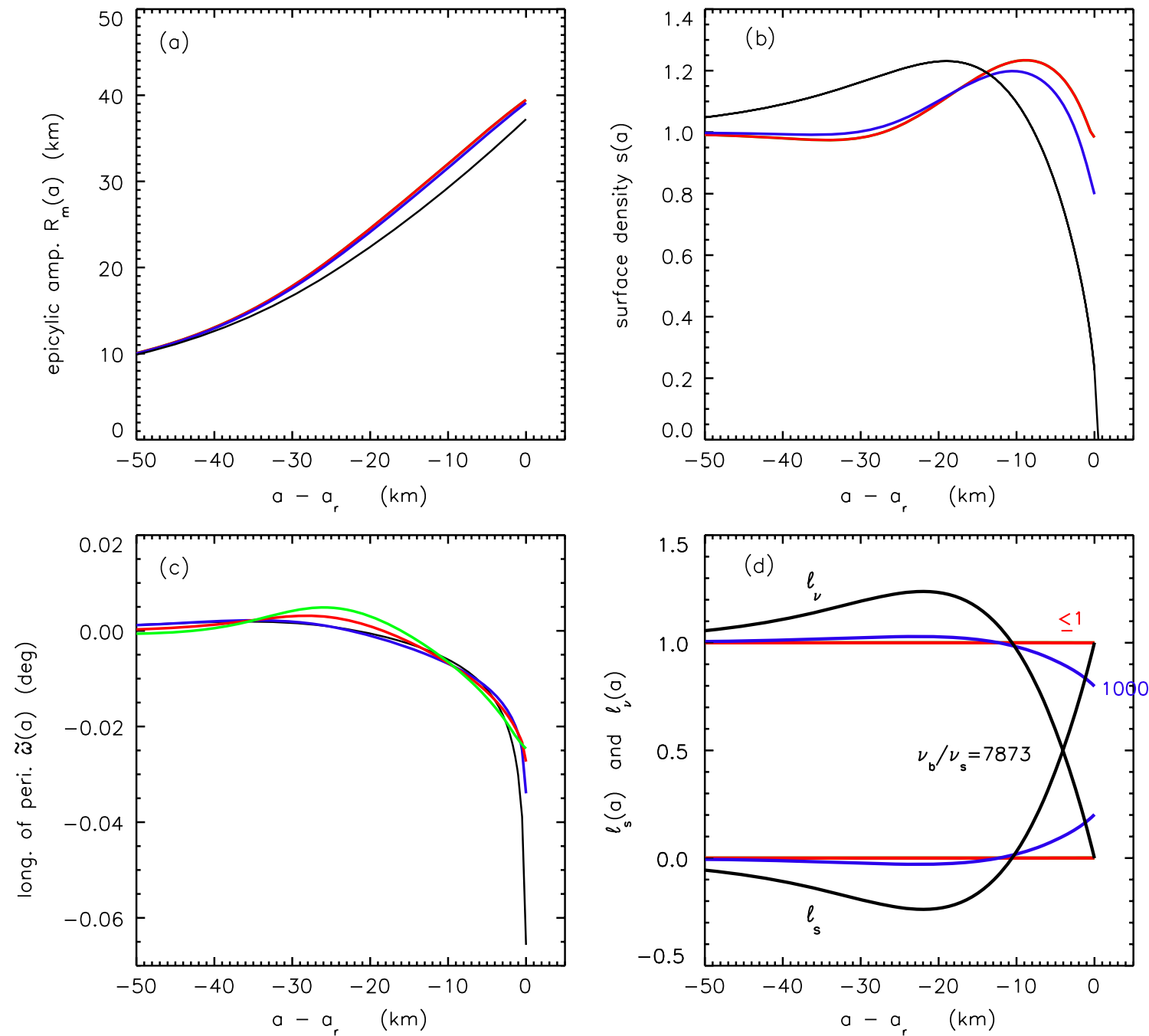

Figure 6. Epicyclic amplitude $R_{m}$ (a) plotted vs. radial distance $a-a_{r}$ from the simulated B ring's outer edge, as well as its fractional surface density $s$ (b), longitude of periapse $\tilde{\omega}(\mathrm{c})$, and the angular momentum luminosities $\ell_{v}$ and $\ell_{s}(\mathrm{~d})$. Shown are the results of three simulations that all have the same undisturbed surface density $\sigma_{\infty}=30 \mathrm{gm} \mathrm{cm}^{-2}$, stability parameter $Q=2$, no drag $\left(C_{d}=0\right)$, and an edge at the nominal resonance where $a=a_{r}$. $N=300$ streamlines are used to model the ring's outermost $w=150 \mathrm{~km}$ with a spatial sampling of $\Delta a=0.5 \mathrm{~km}$. The simulated rings' shear and bulk viscosities all satisfy $v_{s}+v_{b}=100 \mathrm{~cm}^{2} \mathrm{~s}^{-1}$ while having distinct ratios $v_{b} / v_{s}=0$ (green curve), 1 (red), 1000 (blue), and 7873 (black curve, which is the only simulation that satisfies the torque-balance requirement). Note that simulations having $v_{b} / v_{s}=0$ and $v_{b} / v_{s}=1$ are indistinguishable in (a), (b), and (d), so the green curves are hidden under the red. The torque that the satellite exerts on these model rings ranges over $8.8 \times 10^{-5}<\left|T_{s} / T_{G T}\right|=1.5 \times 10^{-4}$.

and with the ring's outer edge stationed at the nominal resonance position at $a=a_{r}$.

But note that the B ring's mean outer edge actually lies about $24 \mathrm{~km}$ exterior to the nominal resonance, and that an outward shift in the resonance position would also result in a larger epicyclic amplitude $R_{m}$ (Section 3.2.2). But that can be compensated for with a larger surface density $\sigma_{\infty}$, which reduces $R_{m}$ (Section 3.2.1). Figure 7 illustrates one possible B ring solution that does achieve a balance of the viscous and satellite torques at the ring's outer edge at $a-a_{r}=24 \mathrm{~km}$, which is where $s \rightarrow 0$ while $\ell_{v} \rightarrow 0$ and $\ell_{s} \rightarrow 1$, and also has the observed epicyclic amplitude of $R_{m} \simeq 45 \mathrm{~km}$ at the ring's edge. This simulation has $\sigma_{\infty}=226 \mathrm{gm} \mathrm{cm}^{-2}, Q=2$, $C_{d}=0$, a total kinematic viscosity of $v_{s}+v_{b}=100 \mathrm{~cm}^{2} \mathrm{~s}^{-1}$, a shear viscosity of $v_{s}=0.00603 \mathrm{~cm}^{2} \mathrm{~s}^{-1}$, and a viscosity ratio of $v_{b} / v_{s} \simeq 1.6 \times 10^{4}$. Note that these simulations depend only on the viscosity ratio $v_{b} / v_{s}$. For instance, when the simulation of Figure 7 is executed again using shear and bulk viscosities that are $\times 10$ larger, the same Figures 7(a), (b), and (d) are obtained, while the longitude of periapse $\tilde{\omega}$ (Figure 7(c)) is larger by $\times 10$, as expected (e.g., Equation (83b)).
Figure 7(b) shows that the simulated ring is also very nonlinear, with $q=0.868$ at its outer edge. This is actually just slightly larger the expected maximum possible value of $q^{\star} \simeq 0.866$, but keep in mind that this $q^{\star}$ threshold is approximate; see just below Equation (67). This large nonlinear parameter also results in significant longitudinal variations in the ring's surface density $\sigma$. These variations are demonstrated in Figure 8, which plots radial profiles of the ring's surface density $\sigma(r, \theta)$ along the satellite's longitude $\theta=\theta_{s}$, as well as along longitude $\theta=\theta_{s} \pm 45^{\circ}$ and $\theta=\theta_{s} \pm 90^{\circ}$. Those curves are calculated via

$$
\begin{gathered}
\frac{\sigma(a, \theta)}{\sigma_{\infty}}=\frac{s(a)}{1-q \cos (\phi+\eta)} \\
\begin{aligned}
\Delta r(a, \theta)= & -a_{r} \simeq a_{r}(x-e \cos \phi) \\
\text { and } \quad \phi(a, \theta) & =m\left(\theta-\theta_{s}-\tilde{\omega}\right) \\
& =\phi^{\star}+m\left[\tilde{\omega}\left(a_{\text {edge }}\right)-\tilde{\omega}(a)\right],
\end{aligned}
\end{gathered}
$$

which may be obtained from Equations (34), (39), and (43), where $\Delta r(a, \theta)=r-a_{r}$ is the radial distance of streamline $a$ at longitude $\theta$ from the nominal resonance $a_{r}$, and $\tilde{\omega}\left(a_{\text {edge }}\right)$ 

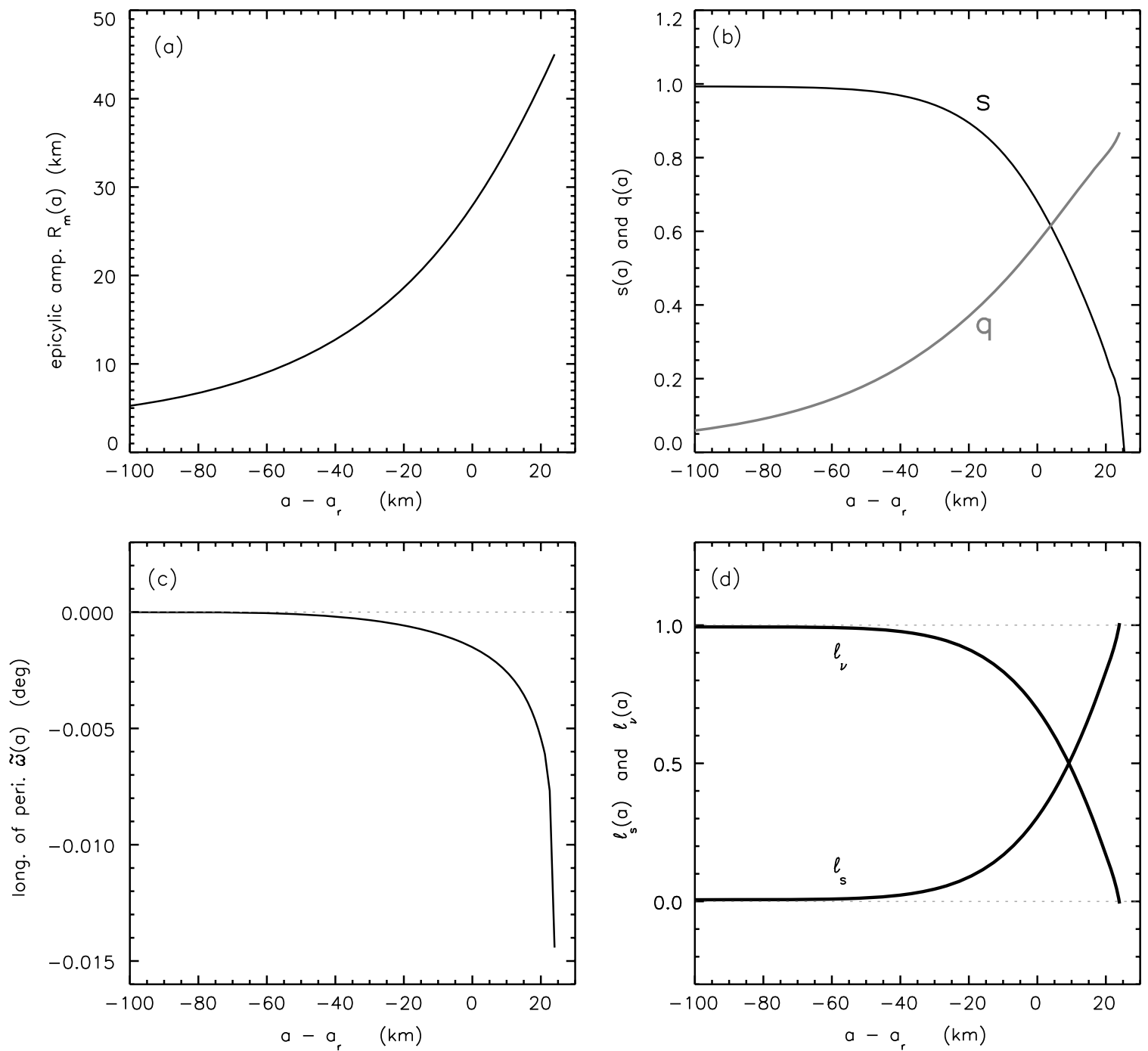

Figure 7. Epicyclic amplitude $R_{m}$ (a), fractional surface density $s$ and nonlinearity parameter $q$ (b), longitude of periapse $\tilde{\omega}$ (c), and the angular momentum luminosities $\ell_{v}$ and $\ell_{s}(\mathrm{~d})$ all plotted vs. radial distance $a-a_{r}$ for a simulated B ring that has an undisturbed surface density $\sigma_{\infty}=226 \mathrm{gm} \mathrm{cm}{ }^{-2}$, stability parameter $Q=2$, a kinematic shear viscosity of $v_{s}=0.00603 \mathrm{~cm}^{2} \mathrm{~s}^{-1}$, kinematic bulk viscosity $v_{b}=100 \mathrm{~cm}^{2} \mathrm{~s}^{-1}$, no drag $\left(C_{d}=0\right)$, and with an outer edge that lies $24 \mathrm{~km}$ beyond the nominal resonance position. $N=300$ streamlines are used over the ring's outer $424 \mathrm{~km}$, so this calculation has a spatial sampling of $\Delta a=1.41 \mathrm{~km}$. The torque that the satellite exerts on the model ring is $\left|T_{S} / T_{G T}\right|=4.2 \times 10^{-5}$.

is the longitude of periapse at the ring's outer edge. Setting the angle $\phi^{\star}=m\left[\theta-\theta_{s}-\tilde{\omega}\left(a_{\text {edge }}\right)\right]=0$ generates a radial profile along the ring-edge's longitude of periapse, while setting $\phi^{\star}= \pm 180^{\circ}$ results in a surface-density profile toward the ring-edge's apoapse. Figure 8 shows that the periapse profile has a large surface density excess at the ring's outer edge, which is due to the satellite's perturbation having shoved ring material inward and compressing the streamlines there. Conversely, the outer edge of the apoapse profile shows a broad low-density shoulder, which is due to the streamlines being more distended there. Since the $|\tilde{\omega}|$ and $|\eta|$ are all small in this model, the intermediate $\phi^{\star}= \pm 90^{\circ}$ profile, which is along longitude $\theta=\theta_{s} \pm 45^{\circ}$, is also where $J \simeq 1$ and $r \simeq a$, so the surface density along this longitude equals its so-called undisturbed surface density $\sigma_{0}(a)$ that the ring has adopted in order in order to achieve a torque balance.

So to summarize the results of this model of a viscous gravitating B ring: if the B ring is indeed a viscous Newtonian fluid, then its edge near Mimas' $m=2$ ILR can only be accounted for when the ratio of the ring's bulk/shear viscosities takes extreme values of $v_{b} / v_{s} \gtrsim 10^{4}$. The ring's bulk viscosity $v_{b}$ is a measure of the friction that results when the ring is compressed radially by the satellite's perturbation. When that is large enough, the shepherding torque exerted by the satellite is then strong enough to counterbalance the ring's viscous torque, thereby confining the ring in the vicinity of the resonance. However, it was a surprise to find that a more conventional model, one having a $v_{b}$ that was comparable or less than $v_{s}$, failed by a wide margin to balance the viscous and satellite torques. Also keep in mind that the ring viscosity $v$ that is inferred from studies of planetary rings (e.g., Tiscareno et al. 2007; Porco et al. 2007) is actually a linear combination of $v_{s}$ and $v_{b}$. So if the preceding scenario is correct, then the ring's $v$ is dominated by its bulk viscosity $v_{b}$, while the ring's shear viscosity $v_{s}$ is negligible in comparison. If this finding is correct, then the B ring can shear freely with little dissipation, while its compressed radial motions are very dissipative.

This is an unexpected finding. Nonetheless, the viscosity requirement $v_{b} / v_{s} \gtrsim 10^{4}$ might be satisfied if the $\mathrm{B}$ ring's compressed regions are so densely packed that the ring particles there are "shoulder to shoulder," with little void space between them. In this case, the ring's volumetric density would be nearly 


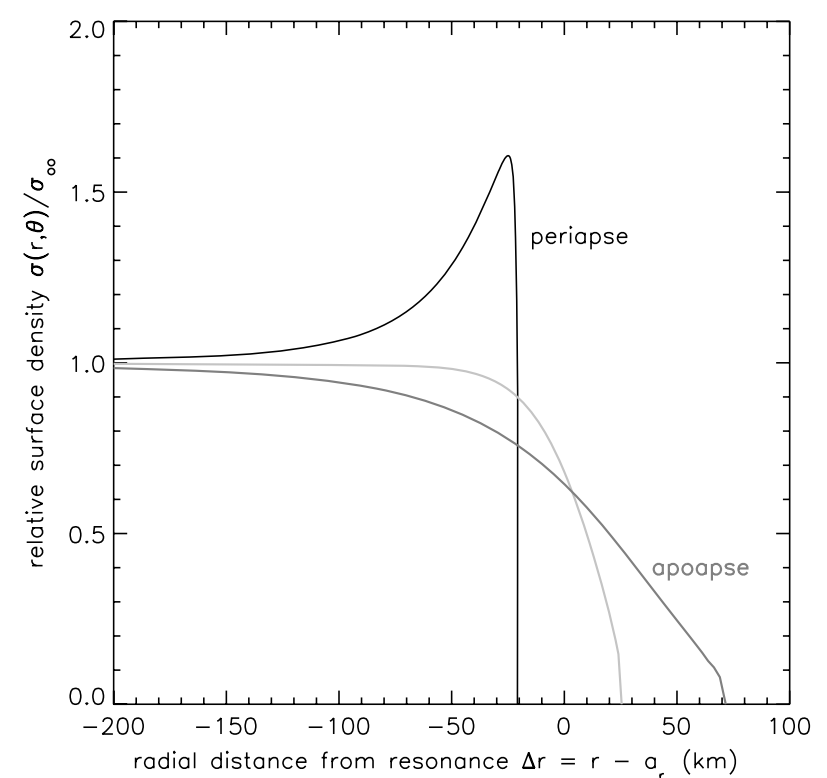

Figure 8. Equation (93a) is used to calculate the ring's relative surface density $\sigma / \sigma_{\infty}$ as a function of semimajor axis $a$ for the viscous B ring model that is described in Figure 7. Equation (93b) is then used to convert that surface density profile into a function of planetocentric distance $r$, which is plotted here vs. distance $\Delta r=r-a_{r}$ from the nominal resonance. These curves give the simulated $\mathrm{B}$ ring's relative surface density along longitude $\theta=\theta_{s}$ (which is along the ring's longitude of periapse), longitude $\theta=\theta_{s} \pm 90^{\circ}$ (along the ring's longitude of apoapse), and along the intermediate longitude $\theta=\theta_{s} \pm 45^{\circ}$.

incompressible, as envisioned by Borderies et al. (1985). This volumetric incompressibility means that as the satellite attempts to compress the ring's streamlines further in the radial direction, ring particles must rise vertically and roll or tumble over each other as they are shoved inward. This could be a very lossy process, one that might satisfy $v_{b} / v_{s} \gtrsim 10^{4}$, since the particles at the ring-edge would effectively experience $2 m$ "avalanches" during each orbit.

However, it is unknown whether the compressed regions in a confined ring-edge are in fact close-packed. And the suggestion that tumbling close-packed ring particles might satisfy $v_{b} / v_{s} \gtrsim$ $10^{4}$ is at this stage mere speculation. Indeed, the reviewer of this paper suggests that the non-Newtonian properties of selfgravitating wakes might instead play an important role here. However, the relevant physics, such as the kinetic theory of Latter \& Ogilvie (2006), or the results of $N$-body simulations (Daisaka et al. 2001; Salo et al. 2001), are not easily adapted to a semianalytic treatment like ours, and so the consequences of non-Newtonian behavior are not explored here. In light of these uncertainties, we also consider an alternate ring confinement mechanism below, to demonstrate that there may be more than one way for a satellite to resonantly confine a sharp-edged planetary ring.

\subsubsection{Drag in a Planetary Ring}

Section 2.4.7 notes that small ring particles are also susceptible to drag forces, such as PR, plasma, and atmospheric drag, and possibly the Yarkovsky effect. Although a drag force tends to have the greatest influence among smaller ring particles, the smaller particles can still communicate the effects of this drag to the larger particles via collisions. This is due to the enhanced eccentricity damping that a smaller particle experiences (Equation (77)); its lower $e$ will then favor collisions with the larger, more eccentric particles. The cumulative effect of these collisions would then resemble a drag force that also acts on the larger particles, too. But this scenario only works if the smaller ring particles are also sufficiently abundant, i.e., if the ring particles' size distribution is sufficiently steep. Obviously, the outcome of this scenario will depend on the particular drag force that is operative in the ring, as well as the particles' size distribution. And this initial study of drag is not prepared to deal with these uncertain details in a rigorous, quantitative way. Nonetheless, the effects of drag can still be assessed qualitatively by using the simple generic drag acceleration that is described Section 2.4.7, and by assuming that there is a single effective drag parameter $C_{d}$ that adequately describes how this drag effects the entire ensemble of ring particles.

When the drag is the dominant source of dissipation, a particle's dissipation function becomes

$$
\psi_{d}=\psi_{\mathrm{drag}}=-\bar{\alpha}_{d r}^{m}+2 \epsilon \bar{\alpha}_{d \theta}^{m}=-\frac{\epsilon C_{d} e}{m-\epsilon}
$$

(see Sections 2.4.7 and 2.5). This drag force also causes the streamlines' longitudes of perihelia to trail behind the satellite's longitude $\theta_{s}$ by an angle $\tilde{\omega} \simeq \psi_{\mathrm{drag}} / m \psi_{s}=-3 C_{d} e / m f_{\epsilon}^{m} \mu_{s}$ (from Equations (29b) and (83b)). The torque $T_{s}$ that the satellite exerts on the ring is $-1 \times$ Equation (87a), so $T_{s} \propto$ $\int_{\text {ring }} \epsilon \sin m \tilde{\omega} d x^{\prime}$ and $\operatorname{sgn}\left(T_{s}\right)=-\epsilon$. Consequently, this drag force also enables a shepherding torque that tends to drive ring particles radially away from the satellite's orbit, since $T_{s}<0$ at an $\epsilon=+1 \mathrm{ILR}$ and $T_{s}>0$ at an $\epsilon=-1$ OLR. And if the shepherding torque is strong enough, it can counterbalance the viscous torque and maintain the ring's edge near the resonance. ${ }^{5}$ This torque-balance is also known as resonance trapping, and it can occur in a wide variety of disk-perturber systems that also have some dissipation, such as dust trapped at a planet's OLR due to PR drag (Roques et al. 1994), and the capture of planetesimals at a protoplanet's OLR due to the solar nebula's aerodynamic drag (Weidenschilling \& Davis 1985; Malhotra 1993; Hahn et al. 1995).

Ring confinement due to drag is illustrated in Figure 9, which is for a B ring that has a surface density $\sigma_{\infty}=265 \mathrm{gm} \mathrm{cm}^{-2}$ far from the resonance, a kinematic shear viscosity of $v_{s}=46 \mathrm{~cm}^{2}$ $\mathrm{s}^{-1}$, and a drag parameter $C_{d}=1.0 \times 10^{-4}$. These parameters were chosen so that the epicyclic amplitude at the ring's outer edge has the observed value of $R_{m}=45 \mathrm{~km}$, and that the ring's viscous torque balances the satellite's torque at a distance of $24 \mathrm{~km}$ beyond the nominal resonance. Keep in mind that Figure 9 represents just one possible solution for the B ring, since other judiciously chosen combinations of ring parameters will also lead to a torque balance at $24 \mathrm{~km}$ beyond the nominal resonance.

Figure 9(c) also shows that when drag is the dominant source of dissipation, then a large longitudinal offset becomes possible, with $\tilde{\omega} \simeq-23^{\circ}$ at the ring's outer edge in this example. But keep in mind that $\tilde{\omega} \propto-C_{d}$, so stronger drag would result in a larger $\tilde{\omega}$. Interestingly, Spitale \& Porco (2006) measured an offset of $\tilde{\omega} \simeq-28^{\circ}$ in Cassini observations of the B ring's orientation, yet Voyager observations revealed no significant offset (Porco et al. 1984).

Figure 10 uses Equations (93) to calculate the ring's relative surface density $\sigma(r, \theta) / \sigma_{\infty}$ as a function of radial distance $r$ along selected longitudes $\theta=\theta_{s}$ (which is along the ring's longitude of periapse), $\theta_{s} \pm 90^{\circ}$ (toward the ring's apoapse),

\footnotetext{
5 Of course, drag forces such as plasma drag, Yarkovsky, etc., can also exert an axisymmetric $m=0$ torque on a ring particle that would cause it to migrate radially. In that case, a sharp ring-edge would instead indicate a balance between the satellite's shepherding torque and the $m=0$ part of the drag torque.
} 

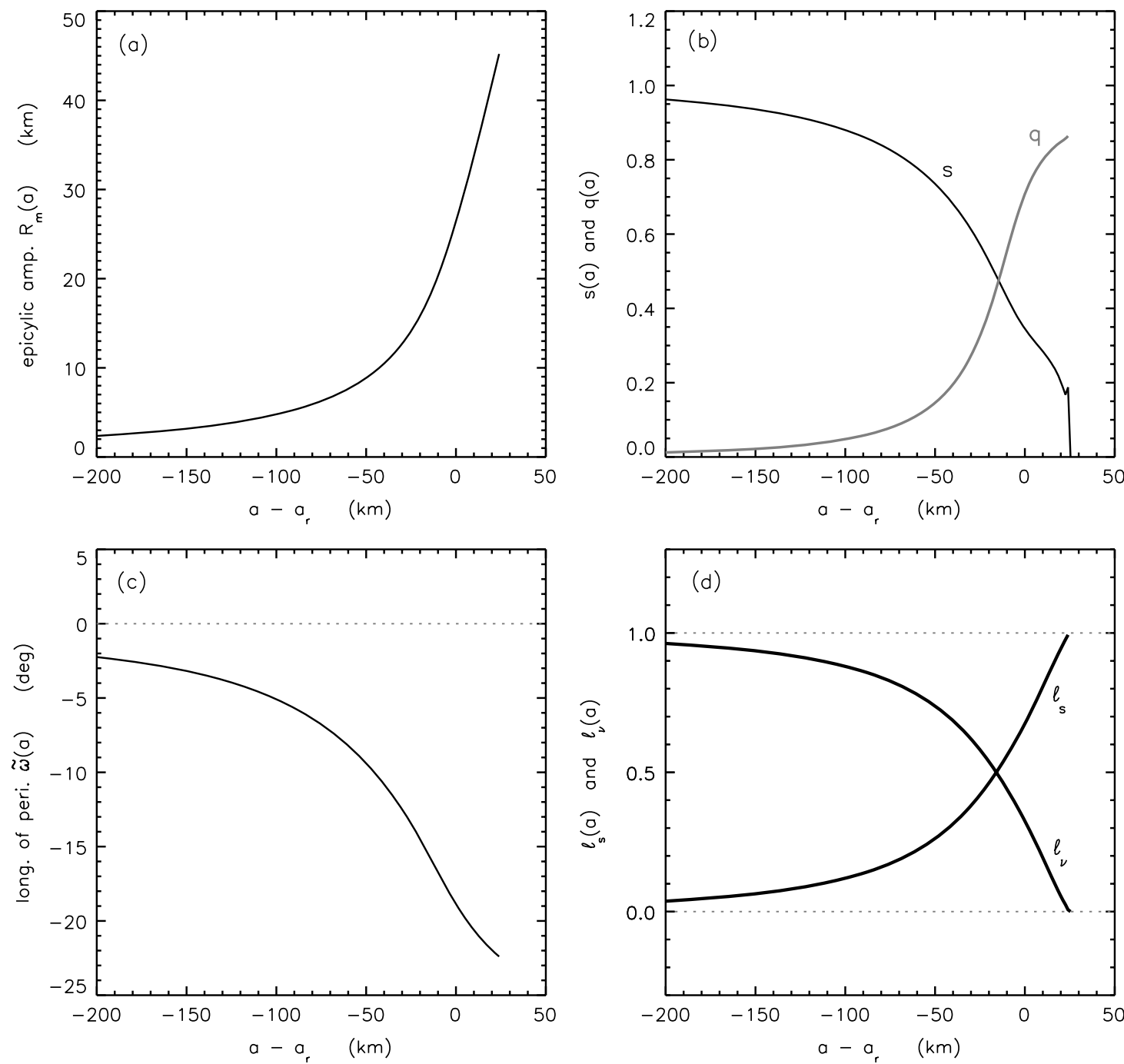

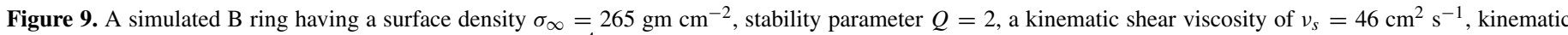

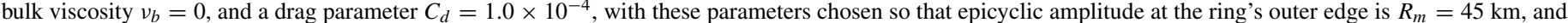

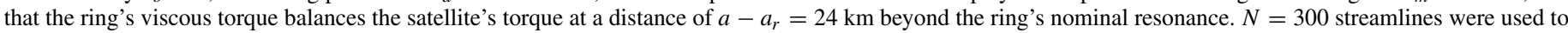
model the ring's outermost $424 \mathrm{~km}$, so the spatial sampling here is $\Delta a=1.41 \mathrm{~km}$. The torque that the satellite exerts on the ring is $\left|T_{s} / T_{G T}\right|=0.32$.

and along the intermediate longitude $\theta_{s} \pm 45^{\circ}$. Again, the model predicts a large surface density enhancement at the ring-edge's periapse, as well as a low surface density shoulder at the ringedge's apoapse.

Evidently, one of this model's main predictions is that the ring-edge's surface density should increase by $\sim 50 \%$ at periapse; see Figures 8 and 10. Presumably, this periapse enhancement would have been seen by the Cassini spacecraft, if not by Voyager. One possible explanation for this nondetection can be found in Porco et al. (2008), which describes a sophisticated photometric model of a swarm of ring particles that are illuminated by the Sun and Saturn, and also imaged by a nearby spacecraft. That model predicts that the optical surface brightness $I / F$ of Saturn's main rings should saturate when the ring's optical depth exceeds about 0.3. Since the optical depth of the outer B ring exceeds that threshold (Colwell et al. 2007), this $I / F$ saturation could account for the absence of any detection of the expected periapse enhancement of the ring-edge's surface density. However, the ring should be less optically thick when observed at longer wavelengths, so the anticipated periapse enhancement might be detectable when observing the ring during a radio occultation.
The main purpose of this section is to illustrate how other forms of ring dissipation might enable a satellite to confine a ring at a Lindblad resonance. For instance, drag forces might play a role in the confinement of the inner Uranian rings, which do orbit close enough to that planet to experience a drag with that planet's extended upper atmosphere (Goldreich \& Porco 1987). However, it should be noted that the differential particle size distribution in Saturn's B ring varies as $d N(s) \propto s^{-q}$, where $s$ is the particle radius and $q \simeq 2.75$ (French \& Nicholson 2000). Consequently, the $\mathrm{B}$ ring's differential mass distribution varies as $d M(s) \propto s^{3-q \simeq 0.25}$, which is weighted toward the larger particles, so the B ring is probably immune to the effects of drag forces.

\subsubsection{The Satellite's Torque}

As was noted above in Section 3.2.4, all of the B ring models that are described in Sections 3.2.1-3.2.4 and Figures 1-5 failed to find a solution that balances the satellite's torque on the ring against the ring's own viscous torque. This was rather surprising since most of those models were constructed (or so we thought) so that the satellite's torque would counterbalance the viscous 


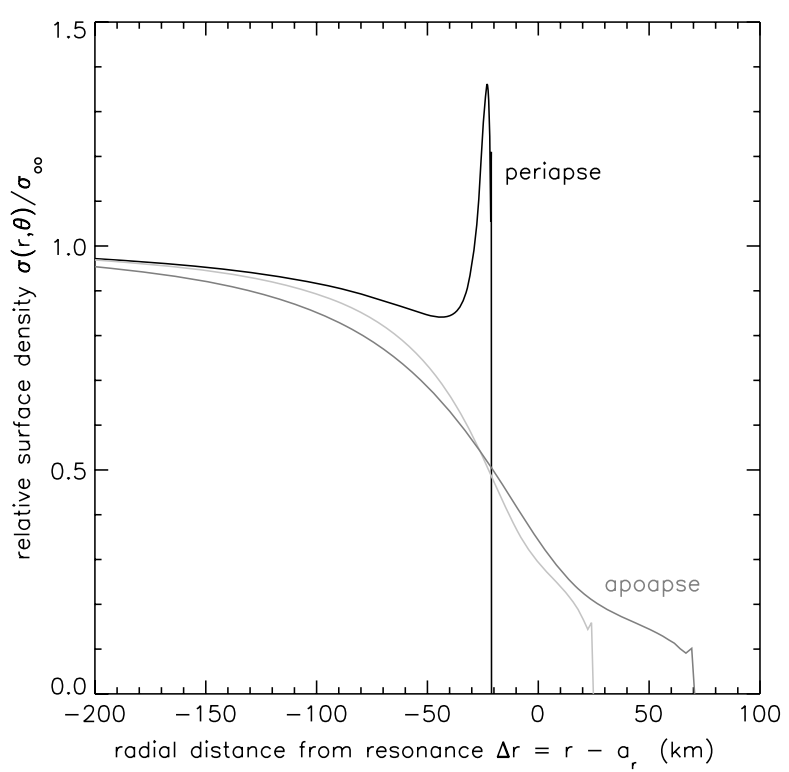

Figure 10. Equations (93) are used to calculate the ring's relative surface density $\sigma(r, \theta) / \sigma_{\infty}$ as a function of radial distance $\Delta r=r-a_{r}$ from the nominal resonance for the model $\mathrm{B}$ ring that is described in Figure 9. These curves give the simulated ring's relative surface density along the ring-edge's longitude of periapse, its longitude of apoapse, and at an intermediate longitude. Note also the small bumps seen at the rightmost part of these curves. They are due to the small, marginally resolved surface density excess that is barely seen to the right in Figure 9(b). That bump is due to the pressure drop that the outermost streamline experiences (e.g., Equation (57)), and it disappears when the ring is pressureless with $Q=0$.

torque. The maximum torque that a satellite can exert on material orbiting at its $m$ th Lindblad resonance is

$$
T_{\mathrm{GT}}=-\frac{\epsilon m \pi^{2} \sigma_{\infty} a^{2}\left(\Psi_{m}^{s}\right)^{2}}{|\mathcal{D}|}=-\frac{\epsilon \pi\left(f_{\epsilon}^{m} \mu_{s}\right)^{2}}{3(1-\epsilon / m)} \mu_{d} M_{p}(a \Omega)^{2}
$$

(Goldreich \& Tremaine 1978, 1982). The viscous torque is the ring's viscous angular momentum luminosity, $\mathcal{L}=$ $3\left(v_{s} / a^{2} \Omega\right) \mu_{d} M_{p}(a \Omega)^{2}$ (see just below Equation (88)), so one might expect a torque-balance to be possible when $\left|T_{\mathrm{GT}}\right|>T_{\nu}$, or when

$$
v_{s}<\frac{\pi\left(f_{\epsilon}^{m} \mu_{s}\right)^{2}}{9(1-\epsilon / m)} a^{2} \Omega,
$$

which evaluates to $v_{s}<145 \mathrm{~cm}^{2} \mathrm{~s}^{-1}$ for Mimas' $m=2$ ILR. Thus, a torque-balance at the B ring's outer edge should seemingly be possible if the B ring's shear viscosity were comparable or less than that inferred for the A ring. However, the following review of the derivation of the Goldreich-Tremaine torque formula, Equation (95), will show why it can overestimate by a large margin the torque that Mimas exerts at a ring's outer edge.

The easiest way to derive Equation (95) is to consider a dragdominated ring whose dissipation function is Equation (94); that scenario was also considered in Meyer-Vernet \& Sicardy (1987). The real and imaginary parts of a ring particle's equation of motion (81) are

$$
\sin m \tilde{\omega}=\frac{\psi_{d}}{\psi_{s}} \quad \text { and } \quad \cos m \tilde{\omega}=-\frac{\epsilon d e+\bar{\alpha}_{c r}^{m}}{\psi_{s}},
$$

where it is assumed that $\bar{\alpha}_{c r}^{m}$ is real. Squaring and summing Equations (97) also shows that $\left(\epsilon e d+\bar{\alpha}_{c r}^{m}\right)^{2}+\psi_{d}^{2}=\psi_{s}^{2}$, where $\psi_{d}$ is Equation (94) and the $\bar{\alpha}_{c r}^{m}$ is the acceleration on a particle that is due to gravity plus pressure. If that $\bar{\alpha}_{c r}^{m}$ term can be neglected, then the above expressions provide the particle's orbit elements in a drag-dominated ring, which are

$$
e(x) \simeq \frac{\left|\psi_{s}\right|}{\sqrt{x^{2}+c_{d}^{2}}} \quad \text { and } \quad \sin m \tilde{\omega}(x) \simeq-\frac{c_{d}}{\sqrt{x^{2}+c_{d}^{2}}}
$$

where $d(x) \simeq x$ (see Equation (82)) and with $c_{d}=C_{d} /(m-\epsilon)$. The satellite's radial torque density is Equation (85), so the total torque that the satellite exerts on the ring is the integral

$$
\begin{aligned}
T_{s} & =\int_{\text {ring }} \frac{\partial T_{s}}{\partial a} d a \\
& =m \pi \sigma_{\infty} a^{3} \Psi_{s}^{m} \int_{\text {ring }} e s \sin (m \tilde{\omega}) d x^{\prime} \\
= & -\epsilon m \pi \sigma_{\infty} a^{3} \Psi_{s}^{m} \psi_{s} c_{d} \int_{-\infty}^{\infty} \frac{d x}{x^{2}+c_{d}^{2}} \\
= & -\frac{\epsilon m \pi^{2} \sigma_{\infty} a^{2}\left(\Psi_{m}^{s}\right)^{2}}{|\mathcal{D}|}=T_{\mathrm{GT}}
\end{aligned}
$$

when it is assumed that the ring has a constant surface density $(s=1)$ that extends everywhere. But if the ring instead had a sharp edge at the resonance, then the satellite's torque would be half that.

The integrand in Equation (99b) indicates that the satellite's torque on the ring is exerted over a fractional radial scale $|x|_{\text {torque }} \sim c_{d}$, which makes sense since this is where the streamlines' $e$ and $|\tilde{\omega}|$ are maximal (Equations (98)). But keep in mind that Equations (98) also ignored the ring's internal forces $\bar{\alpha}_{c r}^{m}$, which Section 2.4.2 showed to be important in the nonlinear zone whose radial extent is $x_{\mathrm{NL}}=\sqrt{\left|\psi_{s}\right|}$ from the resonance (Equation (44)). Those internal ring forces also tend to inhibit streamline-crossing, which they achieve by reducing the streamline's eccentricities below that given in Equation (98). This in turn reduces the satellite torque to something less than Equation (99b). But this torque reduction should still be insignificant whenever $x_{\mathrm{NL}} \ll|x|_{\text {torque }}$, i.e., when the revised drag parameter satisfies $c_{d} \gg \sqrt{\left|\psi_{s}\right|}$. If, however, this criterion is not satisfied, then the Goldreich-Tremaine torque formula, Equation (95), will overestimate the satellite's torque on the ring.

Of course, the dissipation in the ring models of Sections 3.2.1-3.2.4 and Figures 1-5 was due to viscosity rather than drag. However, the preceding discussion suggests that Equation (95) will provide a reliable estimate of the satellite's torque whenever $\Delta a_{\tilde{\omega}}$, which is defined here as the radial distance over which the satellite substantially excites the streamlines' $\tilde{\omega}$, satisfies $\Delta a_{\tilde{\omega}} \gg \Delta a_{\mathrm{NL}}$ where $\Delta a_{\mathrm{NL}}=\sqrt{\left|\psi_{s}\right|} a_{r}$ is the width of the nonlinear zone in physical units. Note that the width of Mimas' $m=2$ ILR is $\Delta a_{\mathrm{NL}} \simeq 20 \mathrm{~km}$ (Section 2.4.2), while Figures 4 and 5 show that these viscous ring models have $\Delta a_{\tilde{\omega}} \lesssim 10 \mathrm{~km}$. Consequently, the satellite's torque is reduced below Equation (95), which explains those models' difficulty in achieving a torque balance at the ring's outer edge.

The torque $T_{s}$ that the satellite exerts on each model ring is also reported in all of the figure captions. Those captions show that the ring's internal forces-gravity and pressurereduce the satellite's torque below Equation (95) by 3-7 orders of magnitude. This explains why the viscous ring model of Figure 7 requires a viscosity ratio $v_{b} / v_{s} \sim 10^{4}$, which also boosts the satellite's torque by that factor, in order to achieve 
a torque-balance at the B ring's outer edge. The exception of course is the drag-dominated ring of Figure 9. In that model, the satellite's torque is only about three times smaller than $T_{\mathrm{GT}}$, with the greater torque efficiency being due to the drag's ability to excite larger $\tilde{\omega}$ across a wider radial span in the ring such that $\Delta a_{\tilde{\omega}}>\Delta a_{\mathrm{NL}}$.

\subsubsection{Assessing the Ring's Internal Forces}

The relative importance of the various accelerations that a ring particle experiences due to ring gravity, pressure, viscosity, etc., is assessed by dividing the real part of the complex equation of motion (81) by the satellite's forcing function $\psi_{s}$, which yields

$$
\left(\mathcal{A}_{c p}+\mathcal{A}_{g}+\mathcal{A}_{p}\right) \cos (m \tilde{\omega})-\left(\mathcal{A}_{v}+\mathcal{A}_{d}\right) \sin (m \tilde{\omega})+1 \simeq 0
$$

where $\mathcal{A}_{c p}=\epsilon d e / \psi_{s}, \mathcal{A}_{g}=\mathfrak{R} e\left(\bar{\alpha}_{g r}^{m}\right) / \psi_{s}, \mathcal{A}_{p}=\mathfrak{R} e\left(\bar{\alpha}_{p r}^{m}\right) / \psi_{s}$, $\mathcal{A}_{v}=\Re e\left(\psi_{v}\right) / \psi_{s}$, and $\mathcal{A}_{d}=\Re e\left(\psi_{\mathrm{drag}}\right) / \psi_{s}$, where $\psi_{v}$ and $\psi_{\text {drag }}$ are Equations (92) and (94). Note that Equation (100) is only approximately true because the accelerations appearing in the equation of motion (81) are complex, yet Equation (100) neglects their smaller imaginary parts. The quantities $\mathcal{A}_{g}, \mathcal{A}_{p}$, $\mathcal{A}_{v}$, and $\mathcal{A}_{d}$ represent the strength of the $m$ th component of a particle's acceleration due to ring gravity, pressure, viscosity, and drag, all in units of the satellite's forcing $\psi_{s}$, while $\mathcal{A}_{c p}=$ $\epsilon e d / \psi_{s}$ is the relative strength of the centrifugal and Coriolis accelerations that the central planet exerts on the particle due to its noncircular motions.

The relative accelerations $\mathcal{A}$ are shown in Figure 11 for the two models that achieved a torque-balance at the ring's outer edge: the model described in Section 3.2.5 and Figures 7 and 8 that invokes an extreme viscosity ratio $v_{b} / v_{s} \gg 1$ to achieve its torque-balance (see Figure 11(a)), and the model of Section 3.2.6 and Figures 9 and 10 that relies on drag to balance the ring-satellite torques (Figure 11(b)). Both models show that the $\mathrm{B}$ ring's internal accelerations are small interior to the ring's outermost $\sim 40 \mathrm{~km}$. There, the particle's motion balances the satellite's forcing against the central planet's centrifugal/ Coriolis forces, i.e., $\mathcal{A}_{c p} \simeq-1$ since $|\tilde{\omega}| \ll 1$, which is equivalent to the single particle solution, Equation (29a). Figure 11 also shows that, in the model B ring's outermost $\sim 40 \mathrm{~km}$, the ring's gravity is the dominant internal ring-force whose strength is comparable to the satellite's forcing and the central planet's centrifugal/Coriolis forces. Those curves show that the acceleration due to ring pressure is small everywhere except at the ring's outer edge, whose effects there are only marginally resolved in these models (see Section 3.2.3). Figure 11 also shows that viscosity has no direct effect on the ring's epicyclic amplitude; instead, its influence enters indirectly via the torquebalance Equation (89). Figure 11(b) also illustrates how strong the drag force must be if it is indeed responsible for a torquebalance at the $B$ ring's outer edge.

Lastly, recall Equation (82), which shows that the $m=0$ component of the ring's conservative accelerations, $\alpha_{g r}^{0}+\alpha_{p r}^{0}$, can displace the location of the resonance, which is the site where $d(x)=0$. It turns out that this displacement is quite small-too small to be resolved in the models considered here. However, that displacement is easily inferred from a linear interpolation of the $d\left(x_{i}\right)$ data that the streamline model generates. For instance, an interpolation of the data generated by the two $\mathrm{B}$ ring models in Figure 11 shows that the ring's internal forces displaces Mimas' $m=2$ ILR inward about $15 \mathrm{~m}$. That small displacement is due to ring gravity, since the ring pressure is negligible there. viscous $B$ ring model

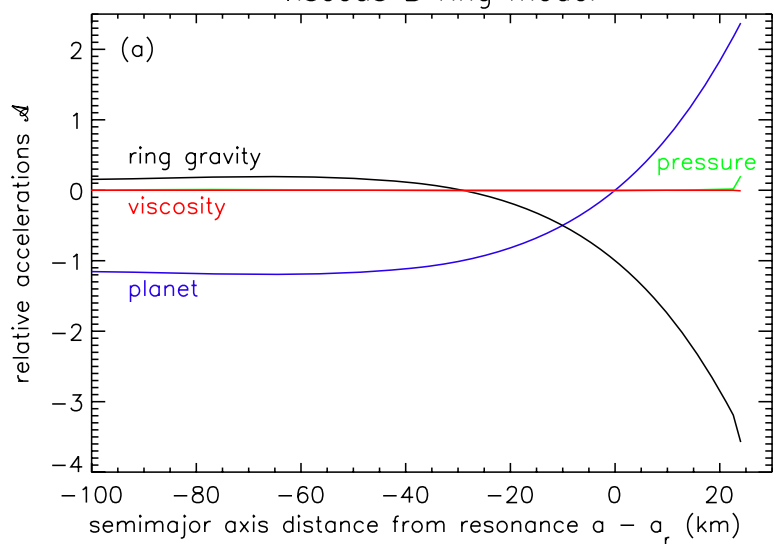

model $B$ ring with drag

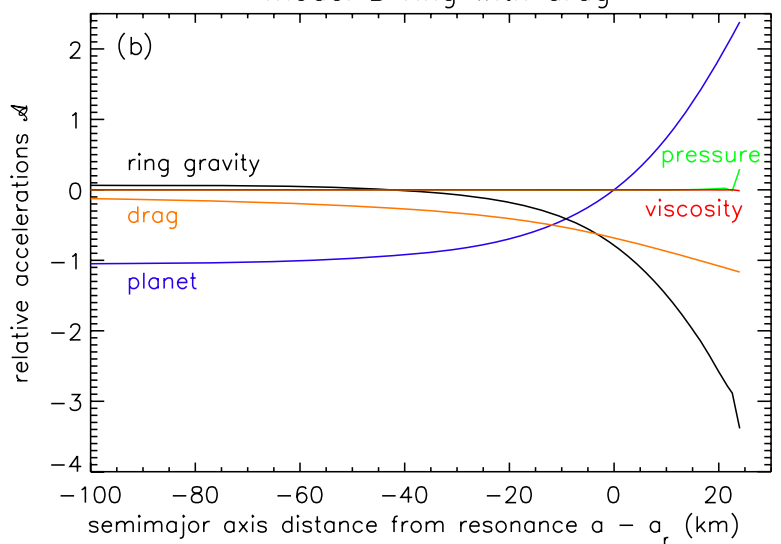

Figure 11. Relative accelerations that a ring particle experiences due to ring gravity $\mathcal{A}_{g}$ (black curve), pressure $\mathcal{A}_{p}$ (green curve), viscosity $\mathcal{A}_{v}$ (red curve), drag $\mathcal{A}_{d}$ (orange curve), and $\mathcal{A}_{c p}$, which is the acceleration that the central planet exerts on the particle; see Section 3.2.8 for details. The upper figure is for the model reported in Figures 7 and 8 that achieved its torque-balance at the ring's outer edge via an extreme viscosity ratio $v_{b} / v_{s}=8473$. The lower figure is for the model described in Figures 9 and 10, which relies on drag to enable a torque-balance.

\section{DISCUSSION}

\subsection{Heating the Ring's Outer Edge}

Another issue that merits consideration is the viscous heating of the ring's outer edge (e.g., Borderies et al. 1982), which might be important when collisions are the dominant source of viscosity, since $v \propto c^{2}$ in this case. To assess this heating, one has to calculate the rate at which the ring's dissipative forces do work on each ring particle. That quantity is probably positive, because the viscous delivery of orbital energy to the ring's outer edge likely exceeds the rate at which the satellite withdraws orbital energy from the ring edge, resulting in a dynamical heating of the ring-edge (Borderies et al. 1982). Of course, other processes also tend to cool the ring particles' random velocities, such as dissipative collisions that convert impact energies into thermal heat, mechanical grinding and fracturing of ring particles, and thermal radiation. Those other cooling mechanisms have not yet been considered, but do need to be included in the ring's energy-balance equation, which could be used to relate the ring's viscosity $v(a)$ to the ring particles' dispersion velocity $c(a)$. But many of the terms in that equation will be difficult to quantify, and will be deferred to a follow-up study. One might have to resort to an order-of-magnitude type analysis, or perhaps settle for an upper limit on the heating that occurs at the ring's edge. However, upcoming Cassini 
observations of the rings during the 2009 August equinox will be helpful here, since that is when the Sun will pass through the ring plane. If the ring-edge is dynamically hot and thick, then the shadows it will cast onto the ring-plane should be observable, which would allow one to infer the ring-edge's vertical scale height $h$ and the ring particles' dispersion velocity $c=h \Omega$ there.

\subsection{Future Applications}

The outer edge of Saturn's A ring is maintained by an $m=7$ ILR with the coorbital satellites Janus and Epimetheus (Porco et al. 1984), whose semimajor axes differ only by about $0.03 \%$ (Jacobson et al. 2008). These satellite's mutual attractions cause their orbits to swap about every four years (Yoder et al. 1983), such that only one coorbital appears to have its $m=7$ ILR in the A ring at any instant of time (J. N. Spitale et al. 2009, in preparation). One might be tempted to use this streamline model to calculate the ring's response to each individual satellite, and then to superimpose the model outputs to obtain the ring's total response to both satellites. However, this might not lead to reliable results, due to the satellites' time-varying orbits. Because the torque that they exert on the A ring changes periodically with time, the outer A ring never experiences a balance of the viscous and satellite torques at any given instant, and so the model's torque balance Equation (89) does not apply here. Instead, a time-average of the outer A ring's viscous torque should be balanced against the time-averaged torque that the coorbitals exert on the ring. However, the calculation of those time-averaged torques is subtle, and will be saved for a followup study of the A ring.

The streamline model can also be used to study narrow eccentric ringlets, but with some revision. For instance, if ringlets are maintained by small unseen shepherd satellites, then the impulse approximation should be used to calculate the torque that they exert on the nearby ringlet. Also, the differential precession that is due to planetary oblateness, which is important in a ringlet system, will also need to be accounted for in a revised version of the streamline model.

Lastly, the streamline model can also be modified so that it can simulate linear as well as nonlinear spiral density waves. Spiral waves transport angular momentum through a ring via the tangential accelerations that particles experience due to ring gravity and pressure. Those terms are negligible in this study of a nearly peri-aligned B ring, but their inclusion will be needed in order to handle the spiral waves' angular momentum transport. Those and other related problems will be considered in followup studies of planetary rings.

\section{SUMMARY OF RESULTS}

The preceding describes a model of a broad, sharp-edged planetary ring that is confined by a satellite's $m$ th Lindblad resonance. This model utilizes the streamline formalism of Borderies et al. (1982, 1985), which makes the calculation of the ring's internal forces - ring gravity, pressure, and viscosityquite tractable. The model also includes a simple prescription for handling the drag forces, such as plasma, Yarkovsky, atmospheric, and/or PR drag, that small ring particles might experience. The model's main inputs are the ring's surface density $\sigma_{\infty}$, the ring particle's dispersion velocity $c$, the ring's kinematic shear and bulk viscosities $v_{s}$ and $v_{b}$, and a dimensionless drag coefficient $C_{d}$. This streamline model solves a nonlinear form of Newton's second law of motion to obtain the streamlines' orbital eccentricities $e(a)$ and longitudes of perihelia $\tilde{\omega}(a)$ as functions of the streamlines' semimajor axis $a$. The model also balances the ring's viscous torque against the satellite's gravitational torque in order to calculate the ring's surface density $\sigma(r, \theta)$ as a function of radius $r$ and longitude $\theta$. That analysis also shows how to use linear and angular momentum fluxes to calculate the effects of viscosity and pressure, both of which are discontinuous at a ring's sharp edge.

The streamline model is then applied to the outer edge of Saturn's B ring, which is maintained by an $m=2$ ILR with the satellite Mimas. A suite of B ring scenarios are examined in order to illustrate how the model outcomes depend upon the ring's physical properties $\sigma_{\infty}, c, v_{s}$, and $v_{b}$, and possibly $C_{d}$, with the main findings listed below.

(1) As one might expect, increasing the ring's surface density tends to decrease the ring-edge's epicyclic amplitude $R_{m}$.

(2) Pressure in the ring is controlled by the ring particle's dispersion velocity $c$, but increases in $c$ are manifest only at the ring's outer edge, which is where the ring's pressure drop is greatest. However, the consequences of that pressure drop are not fully resolved in the models considered here, which have radial samplings of $0.5-2 \mathrm{~km}$.

(3) The magnitude of the B ring's internal forces are compared, and it is shown that the ring's gravity dominates over its other internal forces that are due to pressure and viscosity. In the B ring's outer $\sim 40 \mathrm{~km}$, the gravitational force that the ring exerts on a particle is comparable to the satellite's forcing. However, at sites well interior to the ring's outer $\sim 40 \mathrm{~km}$, ring gravity is relatively small, so the ring particles adopt the familiar single-particle solution $e=\left|\psi_{s} / x\right|$ there.

(4) The ring's viscous torque is controlled by its shear viscosity $v_{s}$, which also governs the rate of the ring's radial spreading. However, the satellite's torque on a viscous ring, which opposes that spreading, is proportional to the angle by which the streamline's periapse lags behind the satellite's longitude. That lag angle is a linear combination of the ring's shear $v_{s}$ and bulk $v_{b}$ viscosities, so the satellite's torque on the ring is sensitive to the sum of those viscosities. Interestingly, a conventional ring model that has a bulk viscosity that is comparable or less than the shear viscosity fails, by a very wide margin, to balance the satellite's torque against the ring's viscous torque, so that scenario fails to account for the B ring's sharp edge near a resonance. Nonetheless, increasing $v_{b}$ does strengthen the satellite's torque on the ring, and models show that a torque balance becomes possible if $v_{b} / v_{s} \sim 10^{4}$. In other words, the ring particles' shearing tangential motions must be relatively free of frictional dissipation, while the ring particles' compressed radial motions must result in heavy frictional dissipation. Such a scenario might be possible if particles in the B ring's compressed regions are confined shoulder-to-shoulder so that there is little void space between the ring particles there, and ring's volumetric density becomes incompressible there. In this case, ring particles must rise and then fall vertically, sliding or perhaps tumbling past one another as the satellite's perturbations drives the ring-edge radially inward and then outward. This periodic avalanche of ring particles could be quite lossy, possibly resulting in a very large bulk viscosity $v_{b}$. However, it is unclear whether the $\mathrm{B}$ ring edge actually behaves in this way, and whether its viscosity can satisfy this remarkable requirement of $v_{b} / v_{s} \sim 10^{4}$, so this finding is speculative. Note also that current ring observations only provide measurements of the combined viscosity $v_{s}+f v_{b}$ where $f \sim \mathcal{O}(1)$, so the ratio $v_{b} / v_{s}$ in a perturbed planetary ring-edge is actually unknown. 
(5) Drag forces provide an alternative means of boosting the satellite's torque on the ring. A generic drag force is considered, one that is proportional to a ring particle's noncircular velocity, and its main effect is to damp the particle's eccentricity while causing its longitude of perihelia $\tilde{\omega}$ to trail behind the satellite's longitude. Since the satellite's torque on the particle is proportional to $\sin (m \tilde{\omega})$, this allows the satellite to torque a wide annulus in the ring, which also makes a torque-balance at the ring-edge favorable. However, this torque-balance due to drag can only be effective if the ring particles' mass distribution is dominated by small particles, which is probably not the case for Saturn's B ring.

(6) The torque that the satellite exerts on the entire ring is calculated for a variety of viscous B ring models, and it is shown to be 3-7 orders of magnitude smaller than that anticipated by the Goldreich-Tremaine torque formula $T_{\mathrm{GT}}$ (Goldreich \& Tremaine 1978, 1982). This is due to the ring's self-gravity, which suppresses the streamline's eccentricities in the nonlinear zone that is near the resonance, which also reduces the satellite's torque there. However, a drag-dominated ring can experience a much larger torque. This is due to the streamlines' trailing longitudes of perihelia $\tilde{\omega}$, which communicates the satellite's torque beyond the nonlinear zone, and can result in a torque that is comparable to $T_{\mathrm{GT}}$.

(7) The outer edge of the B ring could lie as far as $\Delta a \simeq 24 \mathrm{~km}$ beyond Mimas' $m=2$ ILR, which is the site where the ring's viscous torque precisely balances the satellite's torque on the ring. Models of the B ring are adjusted so that the simulated ring-edge's epicyclic amplitude agrees with the observed $R_{m}=45 \mathrm{~km}$ amplitude, and that a torque balance is achieved at distances of $0 \leqslant \Delta a \leqslant 24$ $\mathrm{km}$ beyond Mimas $m=2$ ILR. This requires the outer B ring to have a surface density of $10 \lesssim \sigma_{\infty} \lesssim 280 \mathrm{gm} \mathrm{cm}^{-2}$ in the ring's outermost $\sim 40 \mathrm{~km}$, with the larger surface densities required for rings whose edges lie farther beyond the resonance. A more detailed comparison of models to Cassini observations, which is ongoing, will lead to a more precise measurement of that ring's surface density. Note, though, that the displacement of the ring-edge from the resonance is not due to the ring's internal forces altering the resonance location. For instance, the displacement of the resonance due to ring self-gravity and pressure is tiny, only about $15 \mathrm{~m}$ for the models considered here. But if Mimas had instead been more or less massive, then the B ring's outer edge would then lie interior or exterior to its present location.

(8) Models of the B ring predict that the ring-edge's surface density should be enhanced by $\sim 50 \%$ at the ring's longitude of peripase. The ring's outer edge should also exhibit a low surface density shoulder at the ring's longitude of apoapse. These surface density variations are due to the satellite's perturbations, which compresses the streamlines at periapse and rarefies them at apoapse. It is curious that these periapse enhancements have not been reported in spacecraft observations of the $\mathrm{B}$ ring edge, but this nondetection may be due to a saturation of the ring's surface brightness $I / F$ that is expected to occur when the ring's optical depth exceeds about 0.3 (Porco et al. 2008).

(9) Modifications to the streamline model will also allow its application to other dense planetary rings, such as the outer edge of Saturn's A ring, and the many narrow and sometimes eccentric ringlets that orbit both Saturn and
Uranus. And with additional physics, this model will also provide a useful tool that can be used to simulate nonlinear spiral density waves. Detailed comparisons of models to spacecraft observations of Saturn's rings are ongoing (J. N. Spitale et al. 2009, in preparation), and that activity should yield better estimates of, or else place limits on, the ring's physical parameters $\sigma_{\infty}, c, v_{s}$, and $v_{b}$, and $C_{d}$. Such studies will also lead to a better understanding of the mutual interactions that are exerted in these very interesting ring-satellite systems.

J.M.H.'s contribution to this work was supported by grant NNX07-AL44G issued by NASA's Science Mission Directorate via its Outer Planets Research Program. We also thank Glen Stewart for his review of this work.

\section{REFERENCES}

Borderies, N., Goldreich, P., \& Tremaine, S. 1982, Nature, 299, 209 Borderies, N., Goldreich, P., \& Tremaine, S. 1983, Icarus, 55, 124 Borderies, N., Goldreich, P., \& Tremaine, S. 1985, Icarus, 63, 406 Borderies, N., Goldreich, P., \& Tremaine, S. 1986, Icarus, 68, 522 Borderies, N., Goldreich, P., \& Tremaine, S. 1989, Icarus, 80, 344 Chiang, E. I., \& Goldreich, P. 2000, ApJ, 540, 1084

Colwell, J. E., Esposito, L. W., Sremčević, M., Stewart, G. R., \& McClintock, W. E. 2007, Icarus, 190, 127

Daisaka, H., Tanaka, H., \& Ida, S. 2001, Icarus, 154, 296

French, R. G., \& Nicholson, P. D. 2000, Icarus, 145, 502

Goldreich, P., \& Porco, C. C. 1987, AJ, 93, 730

Goldreich, P., \& Tremaine, S. 1978, Icarus, 34, 240

Goldreich, P., \& Tremaine, S. 1979, AJ, 84, 1638

Goldreich, P., \& Tremaine, S. 1982, ARA\&A, 20, 249

Hahn, J. M., Ward, W. R., \& Rettig, T. W. 1995, Icarus, 117, 25

Hildebrand, F. B. 1956, Introduction to Numerical Analysis, International Series in Pure and Applied Mathematics (New York: McGraw-Hill)

Jacobson, R. A., Spitale, J., Porco, C. C., Beurle, K., Cooper, N. J., Evans, M. W., \& Murray, C. D. 2008, AJ, 135, 261

Jacobson, R. A., et al. 2006, AJ, 132, 2520

Landau, L. D., \& Lifshitz, E. M. 1987, Fluid Mechanics (2nd ed.; Oxford: Elsevier)

Latter, H. N., \& Ogilvie, G. I. 2006, Icarus, 184, 498

Longaretti, P.-Y., \& Borderies, N. 1986, Icarus, 67, 211

Lynden Bell, D., \& Pringle, J. E. 1974, MNRAS, 168, 603

Malhotra, R. 1993, Icarus, 106, 264

Meyer-Vernet, N., \& Sicardy, B. 1987, Icarus, 69, 157

Mosqueira, I., \& Estrada, P. R. 2002, Icarus, 158, 545

Murray, C. D., \& Dermott, S. F. 1999, Solar System Dynamics (Cambridge: Cambridge Univ. Press)

Porco, C., Danielson, G. E., Goldreich, P., Holberg, J. B., \& Lane, A. L. 1984, Icarus, 60, 17

Porco, C. C., Thomas, P. C., Weiss, J. W., \& Richardson, D. C. 2007, Science, 318, 1602

Porco, C. C., Weiss, J. W., Richardson, D. C., Dones, L., Quinn, T., \& Throop, H. 2008, AJ, 136, 2172

Roques, F., Scholl, H., Sicardy, B., \& Smith, B. A. 1994, Icarus, 108, 37

Rubincam, D. P. 2006, Icarus, 184, 532

Salo, H. 1992, Nature, 359, 619

Salo, H., Schmidt, J., \& Spahn, F. 2001, Icarus, 153, 295

Shu, F. H. 1984, Planetary Rings, ed. R. Greenberg \& A. Brahic (Tucson, AZ: Univ. Arizona Press), 513

Shu, F. H., Yuan, C., \& Lissauer, J. J. 1985, ApJ, 291, 356

Spitale, J. N., \& Porco, C. C. 2006, in Lunar and Planetary Institute Conf. Abstracts, Vol. 37, 37th Annual Lunar and Planetary Science Conf., ed. S. Mackwell \& E. Stansbery (Houston, TX: Lunar and Planetary Institute), 2242

Sremcevic, M., Stewart, G. R., Albers, N., Colwell, J. E., \& Esposito, L. W. 2008, BAAS, Division for Planetary Sciences Meeting Abstracts, Vol. 40, \#24.03

Tiscareno, M. S., Burns, J. A., Nicholson, P. D., Hedman, M. M., \& Porco, C. C. 2007, Icarus, 189,14

Toomre, A. 1964, ApJ, 139, 1217

Weidenschilling, S. J., \& Davis, D. R. 1985, Icarus, 62, 16

Yoder, C. F., Colombo, G., Synnott, S. P., \& Yoder, K. A. 1983, Icarus, 53, 431 